\title{
Dinge mit Schrift. Überlegungen zur Inschriftenkultur im mittelalterlichen Genua
}

Genua beeindruckt mit einer besonderen Dichte und Vielfalt an mittelalterlichen Inschriften. ${ }^{1}$ Ihnen wurde bei der Erforschung städtischer Erinnerungskultur in Genua in jüngerer Zeit ein zunehmendes Gewicht beigemessen. Auch jene Objekte wurden verstärkt beachtet, die in Genua bemerkenswert häufig mit Inschriften verbunden sind: Gegenstände der materiellen Kultur, deren Bandbreite von ephemeren Artefakten bis zu Skulpturen reicht. Häufig sind sie in der Funktion von Trophäen präsentiert und damit von Beutestücken, die Vergangenheit deuten und Gedächtnis formen und über die Inschriften hinaus selbst als Medien der Kommunikation im Kontext städtischer Erinnerung wirken. ${ }^{2}$ Das Verhältnis beider, das heißt der Bezug von Inschrift und Objekt, und die Bildlichkeit des dreidimensionalen Dings, als welches sie im Stadtraum Wirkmächtigkeit erreichen, wurden bislang jedoch kaum untersucht. ${ }^{3}$

Die folgenden Überlegungen nehmen ihren Ausgang von Inschriften, die Ereignisse der Stadtgeschichte festhalten oder eine Assoziation dazu herstellen, und die visuell und sprachlich mit einem Objekt verbunden sind, das mehr als nur Inschriftenträger ist. Es handelt sich sowohl um Inschriften auf Objekten als auch um aus zwei materiell distinkten Bestandteilen zusammengesetzte Ensembles. Welche Bedingungen liegen einer derartigen Bild- und Textproduktion zugrunde, und was können die Motivation und der Mehrwert dieser Plurimedialität sein? Objekte ohne Schrift und

1 Die Genueser Inschriften sind, im Vergleich etwa mit jenen in Modena oder Pisa, erst spät in das Blickfeld einer über die Inhalte des Geschriebenen hinaus interessierten kulturhistorischen Forschung geraten. Petrucci 1986, 11, Giovè Marchioli 1994, 274-277 sowie Bottazzi 2012 lenkten in breiterem Zusammenhang das Augenmerk auf Genua; siehe zu den Inschriften im Genueser Kontext auch Dagnino 1987; Müller 2002; Gerevini 2015; Haug 2016.

2 Vgl. generell zu Trophäen Müller 2002, 47-57, zu Pisa Mathews 2012 (die Monographie der Autorin: Conflict, Commerce, and an Aesthetic of Appropriation in the Italian Maritime Cities, 1000-1150, Leiden/Boston 2018, konnte für den Beitrag nicht mehr berücksichtigt werden), zu Genua zuletzt Haug 2016, $193 \mathrm{ff}$.

3 Zur Relation von Bild und Ding Wolf 2015 („,Es wäre an der Zeit, die Bilder ein wenig aus dem übergroßen Druck des Anthropomorphen zu entlassen und ihre Verfasstheit oder agency auch aus ihrer Dinglichkeit oder auf sie hin zu denken“; Wolf 2015, 9), zu Perspektiven der Forschung auch SaurmaJeltsch 2010.- Zu den einzelnen Genueser Monumenten können jeweils nur die wichtigsten Aspekte angesprochen werden, da gerade auf das breite Spektrum dessen, was im 13. und 14. Jahrhundert geschaffen und wahrgenommen wurde, abgehoben werden soll.

Katharina Bolle, Nikolas Jaspert und Marc von der Höh danke ich für die Einladung zu der sehr gewinnbringenden Tagung. Ihnen und besonders Ralf Behrwald, Henrike Haug und Andreas Rehberg bin ich für Diskussionen und Hinweise verbunden; für Informationen zu der Handschrift von Domenico Piaggio danke ich Emanuela Ferro, für die Textlektüre Kristina Hurr.

Ә Open Access. ( 2019 Rebecca Müller, publiziert von De Gruyter. (cc) BY-NC-ND Dieses Werk ist lizenziert unter der Creative Commons Attribution-NonCommercial-NoDerivatives 4.0 Lizenz.

https://doi.org/10.1515/9783110642261-007 
Texte entfalten über ihre Bildlichkeit und ihr Material eine ihnen jeweils spezifische Wirkung und Präsenz. Beide signalisieren im Stadtraum in unterschiedlicher Weise Autorität. Sind sie verbunden, stellt sich die Frage, wie sie sich darin beeinflussen: ob sie sich ergänzen und das eine Medium eine Leerstelle, einen Mangel an Aussagekraft des anderen füllt, oder ob beide sich in dieselbe Richtung verstärken. ${ }^{4}$ Werden Interpretationsspielräume verengt, wenn Inschrift und Objekt gemeinsam Ereignisse deuten, oder werden sie erweitert? Bei zwei der Genueser Inschriften fällt beides in eins, wird die Inschrift als Artefakt zur Trophäe und gleichzeitig zum Gegenstand der städtischen Geschichtsschreibung. Die als medienspezifisch gedachten Aspekte sind hier besonders zu hinterfragen.

Mit Blick darauf, welche Rolle Inschriften für die Kommunikation im Stadtraum zukam $^{5}$ interessiert insbesondere, inwieweit ein Medium die Wahrnehmung des anderen zu lenken vermag und Deutung immer neu konstruiert wird. Der Versuch, anhand einiger Fallbeispiele zumindest die Bedingungen von Wahrnehmung und damit verbundene Deutungsmuster zu erfassen, erscheint grundlegend für eine - an dieser Stelle nur in Ansätzen zu leistende - Verortung des Themas in einer Geschichte der kommunikativen Praktiken. ${ }^{6}$ Damit verbindet sich die Überlegung, inwieweit mit der Differenzierung der Wirkmöglichkeiten so vereinigter Medien sich auch die Rezipienten differenzieren und Identitäten in der Stadt neu oder anders konstruieren, etwa illiteraten Betrachtern Zugänge eröffnet werden, wo der sie vom Wortverständnis ausschließende Part des Textinhalts nur ein Teil der Aussage ist.

Aus dieser Perspektive werden auch andere Aspekte der Tagung berührt, so jener des Verhältnisses zwischen Inschriften, die von kommunalen Amtsträgern in Auftrag gegeben wurden, zu solchen, die sich primär mit einzelnen Familien verbinden. ${ }^{7}$ Einige Objekte führen zu der Überlegung, was sich ändert, wenn ein Inschriftentext in ein anderes Material, eine andere Größendimension, ein anderes Medium übertragen wird. Immer wieder tritt die epigraphische Praxis der römischen Antike in den

\footnotetext{
4 Die Forschung zum Text-Bild-Bezug hat sich ein breites Set an Fragestellungen und Ansätzen erarbeitet; an dieser Stelle sei hier nur auf Kroll 2015 sowie auf den Forschungsabriss für das Mittelalter bei Schellewald 2011 verwiesen. Grundlegend zu einer „iconicity of script“ Hamburger 2014 (Zitat: 2). 5 Petrucci 1985, Debiais 2009 und (mit vergleichbaren Fragestellungen, jedoch mit stärkerer Betonung der sozialen Praxis und der Konstruktion von Identitäten für das fatimidische Ägypten) Bierman 1998; beispielhaft für Pisa von der Höh 2006. Das Spannungsfeld zwischen Ding und Zeichen umreißen unter dem Aspekt der Sichtbarkeit von Schrift grundlegend Strätling/Witte 2006.

6 Vgl. zur Wahrnehmung und Medialität im Mittelalter Kiening 2007, grundlegend zum Begriff der Wahrnehmung aus mediävistischen Perspektiven Bleumer/Patzold 2003. Zum Konzept einer Kommunikationsgeschichte, auch in Abgrenzung zu einer Geschichte der Medien, Depkat 2003. - Wie wichtig die Bedingungen der Wahrnehmung für das Verhältnis von Inschrift und Objekt sind, erhellen auch Analysen von Inschriften auf kleinformatigen, einem kleinen Rezipientenkreis zugänglichen Objekten, siehe etwa Shalem 2016.
}

7 Vgl. hierzu bes. den Beitrag von Flavia De Rubeis in diesem Band. 
Blick und damit die Frage, inwieweit sie paradigmatisch für die Inschriftenkulturen der Kommune wirken konnte.

Bereits für das 12. Jahrhundert lässt sich nachvollziehen, dass im Zuge der Kreuzzüge und der spanischen „Reconquista“ in der Genueser Überlieferung, die beides kommentiert und die Erinnerung daran modelliert, Inschrift und Objekt zusammenwirken und Inschriften jenseits ihres Textinhalts zu begehrten Objekten werden. Wohl drei arabische Inschriften auf Stein wurden in Spanien aus Moschee- oder Grabkontexten entfernt und in Genueser Kirchen eingemauert; ein weiteres, nicht mehr erhaltenes Beutestück, ein Paar bronzene Türflügel aus dem spanischen Almería, ist nur bekannt, weil eine noch im 16. Jahrhundert vorhandene Inschrift seinen Ursprung und die Umstände der Anbringung festhielt. ${ }^{8}$ In der Kathedrale San Lorenzo berichten monumentale gemalte Inschriften von der Einnahme Tortosas (und erwähnten vermutlich weitere Schlachtenerfolge der Genuesen); sie stehen hier neben Historiengemälden des 12. Jahrhunderts, die das Geschehen visuell vermitteln. ${ }^{9}$ Welcher Anteil diesen Texten und Bildern an der Konstruktion von Erinnerung zukommt, hat jüngst Henrike Haug im Zusammenhang mit der städtischen Geschichtsschreibung untersucht. ${ }^{10}$ Auch wenn sich der Beitrag im folgenden auf das 13. und 14. Jahrhundert konzentriert, ist der Umstand festzuhalten, dass man an einen bereits eingeführten Mediengebrauch der jungen Kommune anknüpfte. Diese Tradition konnte nach Bedarf aufgerufen werden. Das 1242 verfasste Gedicht, mit dem der Notar und Diplomat Ursone da Sestri einen Sieg Genuas an der Riviera über ein kaiserlich-pisanisches Heer feiert, belegt dies. Er schreibt: „[D]as berühmte Volk“ - das sind natürlich die Genuesen - „erinnert sich der früheren Triumphe, die es in den verschiedenen Teilen des Erdkreises errungen hat [...] es brennt im Herzen das Raubgut aus Minorca, die Beute aus Almería; Tortosa, das mit Kriegskunst unterworfen. Ihr gemalter Ruhm erstrahlt in der größten Kirche [i.e. San Lorenzo]."11

Die Auseinandersetzungen mit Friedrich II. und mit Pisa rief auch ein Ensemble aus Relief und Inschrift auf, das am Genueser Kommunalpalast angebracht war. Ob das Relief ursprünglich für einen anderen Ort angefertigt wurde und erst später an den 1260 errichteten Palast gelangte, ob es von Beginn an Bauschmuck war, oder ob es im späteren Verlauf des 13. Jahrhunderts entstand, lässt sich nicht entscheiden. Es

\footnotetext{
$8 \mathrm{Zu}$ diesen zuletzt Müller 2016.

9 Calderoni Massetti/Wolf 2012, Bd. 1, 340-343 Scheda 500 (Gianluca Ameri, zu den Inschriften Stefano Riccioni).

10 Haug 2016.

11 Nam genus a magno ducens gens inclita Iano / Antiquas laudes recolit veteresque triumphos / Quos meruit bello diversis partibus orbis / [...] fervet sub pectore praeda Minorcae / Almariae spolium, Tortosaque Marte subacta, / Quarum picta nitet maiori gloria templo. / Dant stimulos, bellique faces, votumque triumphi / Foedera Maiorcae, [...] His stimulis impulsa calens ad proelia fervet, / Atque novos titulos antiquis addere flagrans / Posteritas generosa parat, Ursone da Sestri 1853, Sp. 1755 f.; siehe auch Haug 2016, 267 f. und zum Autor Airaldi 2004, 105-135.
} 
erscheint als zur Konzeption des Palastes gehörig sinnvoll. ${ }^{12}$ Sein Aussehen ist uns in dem aus dem 18. Jahrhundert stammenden Manuskript des Lokalhistorikers Domenico Piaggio überliefert (Abb. 1). Piaggo gibt als Ort „,an der Außenfassade“ an und meint damit vermutlich über der Portikus im ersten Obergeschoß des Palastes. ${ }^{13}$ In den Revolutionsunruhen ging das zwischenzeitlich in den großen Saal des Palastes verbrachte Monument verloren.

Gezeigt war ein Greif - ein Tier, das im 13. Jahrhundert in verschiedenen Medien und Materialien Genua repräsentierte -, der sich einen Adler und einen Fuchs unterwirft. ${ }^{14}$ Piaggio versteht den Adler als Verkörperung des römischen Reichs, und damit als einen Hinweis auf Friedrich den II., und den Fuchs als Symbol für Pisa, beide durch Genua besiegt wie es die Beischrift sagt: „So wie der Greif diese würgt, so überwältigt Genua seine Feinde“ “. ${ }^{15}$ Diese Deutung wird auch in der Forschung vertreten, wobei Clario Di Fabio den Fuchs ausgehend von dessen negativer Charakterisierung etwa im Physiologus als Symbol für den Erzfeind Pisa adaptiert sieht. ${ }^{16}$

$\mathrm{Zu}$ dem Zeitpunkt, als Relief und Inschrift am Kommunalpalast angebracht wurden, waren sowohl die Ikonographie dieser Tierkampfgruppe als auch die Beischrift einem Teil der Bürgerschaft bereits bekannt, denn beides stammt von dem 1192 erstmals beschriebenen städtischen Siegel. ${ }^{17}$ Auf diesem Wachssiegel - das früheste bekannte Exemplar wird in Montpellier aufbewahrt - weisen die Schwingen des Greifen sichelförmig nach oben. Eine spätere Version, deren Entstehung Francesco Gamberoni in die zweite Hälfte des 13. Jahrhunderts datiert, weist hingegen parallel zum Tierkörper geführte Schwungfedern auf, wie sie Piaggio zeigt (Abb. 2).

12 Zur Baugeschichte des Palastes und seiner Nutzung Cavallaro 1992 und Ferrando Cabona 1998, zu seiner kommunalpolitisch programmatischen Ausstattung und dem Relief auch Müller 2002, bes. 86-91 und der Beitrag von Wilfried E. Keil in diesem Band.

13 „Nella facciata esteriore della Casa di S. Giorgio“, Piaggio, MS 1720, Bd. 5, fol. 316r (alte Numerierung fol. 315)

14 Zur Bedeutung des Greifenmotivs für Genua bleibt grundlegend Di Fabio 1989, hier 25 f. zu dem Relief; zu den in Genua verwendeten städtischen Siegeln zuletzt Gamberoni 2004 mit der älteren Literatur.

15 Griphus ut has angit / sic hostes Janua frangit. Unter der Wiedergabe des Reliefs samt seiner Inschrift schreibt Piaggio: „Grifone Insegna antica de Genovesi, che teneva sotto artigli una aquila et una volpe, essendo l'aquila insegna dell'Imperio, come che in quel tempo Federico secondo Imperatore fosse contrario alla Chiesa al sommo Pontefice Innocenzo IV Fiesco Cittadino Genovese, e la volpe Insegna de Pisani speciali nemici de Genovesi“, vgl. Anm. 13.

16 Di Fabio 1989, 41 Anm. 103.

171192 bzw. 1193 schließen die Grafen von Ventimiglia und die Genueser Kommune ein Abkommen, das besiegelt wird $\mathrm{u}$. a. mit einem sigillum comunis Ianue, in quo apparebat forma cuiusdam griffi tenentis inter pedes [...] et vulpem, Puncuh 1996, 413. Das Motiv wurde auch mit einem Hahn anstelle des Adlers variiert, was Probleme in der Deutung aufgeworfen hat, vgl. Gamberoni 2004, 132 f. - Vgl. für die in Paris aufbewahrten Genueser Siegel Douet d‘Arcq 1868, 498 f. 


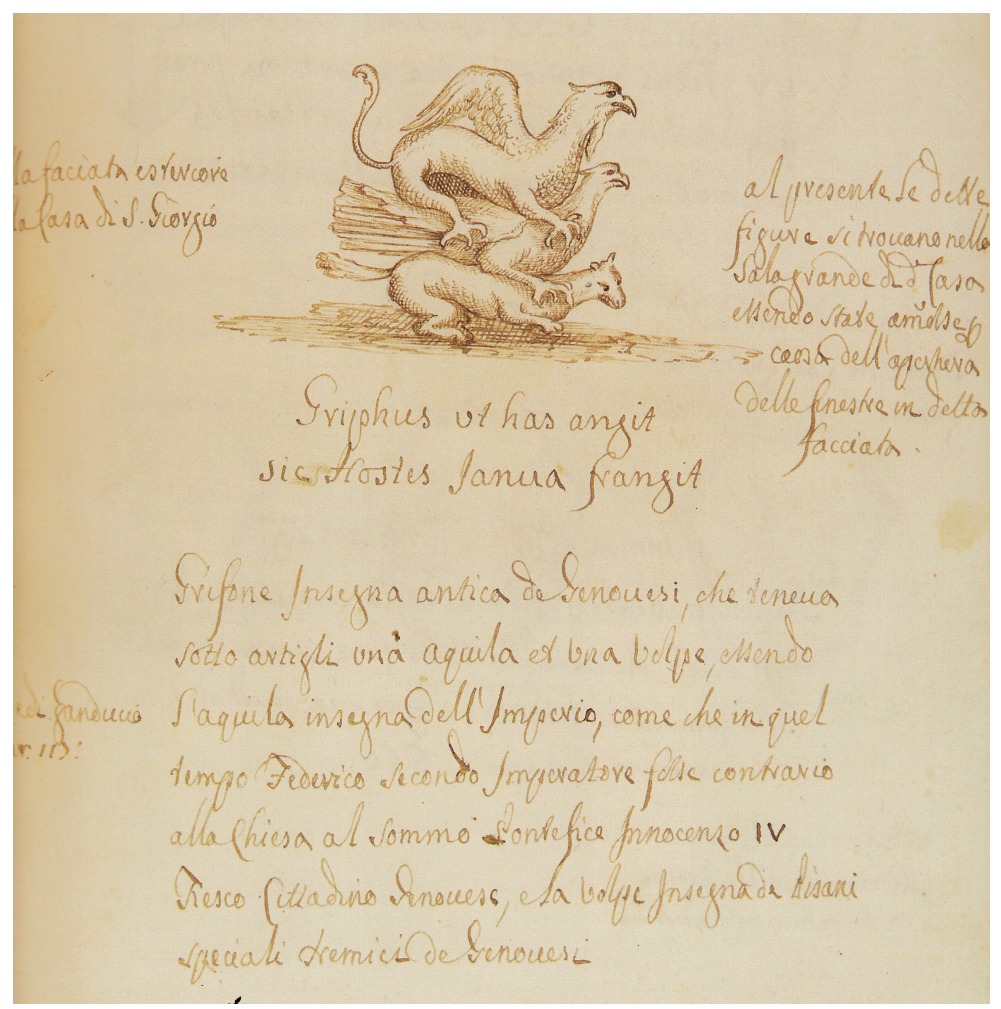

Abb. 1: Domenico Piaggio, Nachzeichnung des Reliefs am Palazzo di San Giorgio in den Epitaphia, sepulcra et inscriptiones cum stemmatibus, marmorea et lapidea, existentia in ecclesiis genuensibus, MS 1720, Genua, Biblioteca Civia Berio.

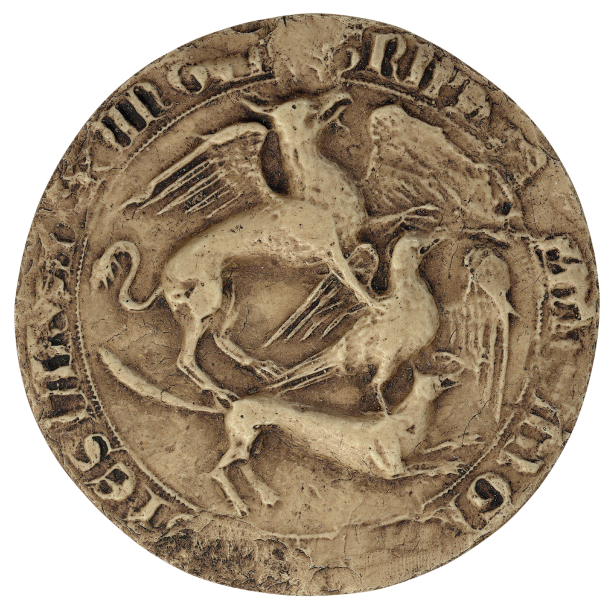

Abb. 2: Siegel der Genueser Kommune (Abguss), zu einer Urkunde vom 7. Juni 1333 gehörig, Paris, Archives nationales. 
Wenn wir dem Autor eine getreue Nachzeichnung zubilligen, dann dürfte diese Version Vorbild für das Relief gewesen sein. Mit diesem Medientransfer war das potentielle Publikum für die Aussage des Siegels maßgeblich erweitert. Ist die Wiedergabe der Inschrift als einer ,Unterschrift‘ durch Piaggio anstelle der umlaufenden Legende des Siegels korrekt, dann war die Provenienz des Motivs nur für die mit dem Siegel Vertrauten offensichtlich und lag die Absicht nicht in einem demonstrativen Verweis auf das Vorbild. Gleichzeitig wurde mit dem geänderten Schriftlayout in der monumentalen Ausführung die auf dem Kopf stehenden Buchstaben vermieden und die Lesefreundlichkeit der ohnehin knappen und markigen Sentenz noch erhöht. Ikonographie und Inschrift scheinen in ihrem Zusammenspiel auf eine breite Verständlichkeit hin angelegt. Der Greif, das ebenfalls in monumentalen Skulpturen verbreitete und damit bekannte Motiv, dominiert das Bild, und der Text setzt mit „GRIPHUS“ ein. Wer das Siegel kannte, dem war die Botschaft auch ohne Text vertraut. Wer es nicht kannte, den konnte das Relief allein über die intendierte Parallelisierung von Tieren und politischen Einheiten noch im Unklaren lassen. Das „so - wie“ des Textes macht auf die notwendige Auslegungsleistung aufmerksam. Allerdings bleiben auch in der Inschrift die Tiere und ihre Bedeutung unbenannt, was alternative und aktualisierte Deutungen der hostes möglich machte. Indem man auf die offensive Rhetorik gerade dieses Siegels zurückgriff, wurde die Sieghaftigkeit Genuas nicht nur zum Thema der Fassade erhoben, sondern das Wirken der kommunalen Vertreter unter dieses Leitmotiv gestellt.

Mehrere Trophäen, die am Kommunalpalast angebracht wurden, bestätigen die Aussage des Reliefs und lösen sie gleichsam ein. Unter ihnen sollen an dieser Stelle nur jene interessieren, die selbst mit Inschriften verbunden waren beziehungsweise bei denen es sich um Inschriften handelt. Die Annalen halten für das Jahr 1256 fest, dass die Genuesen im Zuge der neu aufgeflammten Auseinandersetzungen mit den Pisanern die Ansiedlung Lerici eroberten, die von diesen erst im Jahrzehnt zuvor (1241) besetzt worden war. Lerici war wegen seiner Lage am Golf von La Spezia gegenüber des genuesischen Portovenere von militärischer und handelsstrategischer Bedeutung und ein umkämpfter Vorposten. Der Annalist schreibt:

Sie [die Pisaner] hatten am Eingang der Siedlung im höhergelegenen Teil ein Tor mit zwei Türmen errichtet, von denen einer bereits eine ausreichende Höhe erreicht hatte. Zwischen diesen Türen befand sich ein Stein, an dem ein Ballen gemeißelt war in Form der Stoffe, die aus Frankreich eingeführt werden, und der Stein hatte folgende Inschrift: Stopf dem Genuesen das Maul, brich dem Portoveneresen das Herz, entreiß dem Lucchesen das Stoffbündel [oder: den Sack]. Dieser Stein wurde, nachdem die Siedlung zerstört worden war, nach Genua gebracht. ${ }^{18}$

18 ... in introitu burgi a parte superiori portam cum duabus turribus construxerant quarum una iam erat competentis altitudinis. infra ipsas turres erat quidam lapis in quo erat sculptum torsellum ad formam pannorum qui deferuntur de Francia et erat suprascriptio ipsius lapidis: STOPA BOCA AL ÇENOESE: CLEPA COR ALO PORTONARESE: STREPA TORSELLO ALO LUCHESE qui lapis, burgo dirruto, delatus 
Es handelt sich damit um eine zwischen 1241 und 1256 entstandene pisanische Inschrift, die eine abbrevierte Abbildung einer wichtigen Handelsware des Küstenortes mit Schmähungen verband, die sich gegen Genua und seine Verbündeten richteten, darunter Lucca. Für die Stofffabrikation dieser Stadt war Genua in vieler Hinsicht, etwa mit Blick auf die Rohmaterialien und Farben sowie den Weiterverkauf, ein wichtiger Handelspartner. ${ }^{19}$ Der zitierte Passus der Annalen bildet die einzige Quelle für diese Inschrift, von der wir nicht wissen, wohin sie in Genua gebracht wurde. Hier haben sich ihre Spuren verloren.

Bemerkenswerterweise fand man bei Bauarbeiten im Genueser Palazzo di San Giorgio eine andere Inschrift aus dieser Zeit, die aus Lerici stammen dürfte (Abb. 3). Sie ist gut einen halben Meter breit und in Capitalis rustica auf Marmor ausgeführt. Die Ränder sind abgebrochen, vielleicht Resultat einer gewaltsamen Aneignung, so dass eine Ergänzung und das Verständnis der Inschrift Probleme bereiten..$^{20}$ An dieser Stelle ist als relevant hervorzuheben, dass es sich um eine pisanische Bauinschrift handelt, dass in ihr ein Jacopo Napoleone erwähnt wird, der 1255 Podestà in Pisa war, und dass eine Lokalität „Podio Sale“ genannt ist, die identisch mit einer „Le Sale“ benannten Anhöhe Lericis sein könnte. Lerici selbst wird nicht angeführt, die Herkunft aus diesem Ort muss demnach Vermutung bleiben. ${ }^{21}$

Die Inschrift ist offensichtlich nicht die in den Annalen genannte. Die Parallelen liegen darin, dass in den Auseinandersetzungen mit Pisa Inschriften, deren Wortlaut die Genuesen schmähte beziehungsweise als Bauinschrift pisanisches Territorium markierte, durch die siegreichen Genuesen entfernt, aber nicht zerstört, sondern nach Genua verbracht wurden. Diese Objekte erfuhren vermutlich keine Ergänzungen durch weitere Inschriften, da sie aufgrund der in ihren eigenen Texten getroffenen, genuafeindlichen Aussagen allein durch ihre Anwesenheit in Genua die Niederlage ihrer Verfasser, nämlich der Pisaner bezeugten. Um dies ausspielen zu können, mussten sie nicht als nur Objekte wahrgenommen, sondern als Texte gelesen oder in der Geschichtsschreibung referiert werden. Gleichzeitig wird deutlich, welche Wirk-

fuit in Ianuam (Der Text der Inschrift ist in der Edition hervorgehoben, nicht jedoch in dem zugrundeliegenden Codex). Belgrano/Imperiale di Sant’Angelo 1926, 21; Übersetzung der Verf.; vgl. Müller 2002, $212 \mathrm{f}$.

19 Pessa 2016, bes. 101.

20 Eine Transkription und Ergänzung leistete Ferretto 1904, 141: [Anno MCCLVI]. CIVITAS PISANA D(E) M(EN)SE NOVE(M)BRIS HEDIFICAR(E) / [CEPIT HUNC BURGUM IN] PODIO SALE T(EM)P(O)RE IACOBI NEAPOLEONIS D(E) U/[RBE POTESTATIS ET AND]REE IACOBI D(E) PERUSIO CAPIT(ANEI) PIS(ANI) P(O)P(U)LI ET / [TEMPORE FABRI DE FABRIS] D(E) BON(N)ONIA PIS(ARUM) POTESTATIS ET DICTI / [ANDREE CAPITANEI M]URARI FECIT EXIST(ENT)E VITE POTESTATE / [ILLICIS UGONE CA] RGATTI ET IACOBO D(E) MONTANINO NOT(ARIO) CU(RIE) ...; vgl. auch Silva 1987, 109f. und Müller 2002, 209 Anm. 115 mit Diskussion der Lesung (wobei Vergleiche in der Amtsbezeichnung für eine Ergänzung zu „capitanei pisani populi“ [statt „pisarum“] sprechen, wie es Silva gegen Ferretto vorschlug).

21 Für eine Übersetzung und Diskussion siehe ebd. 209-211. 


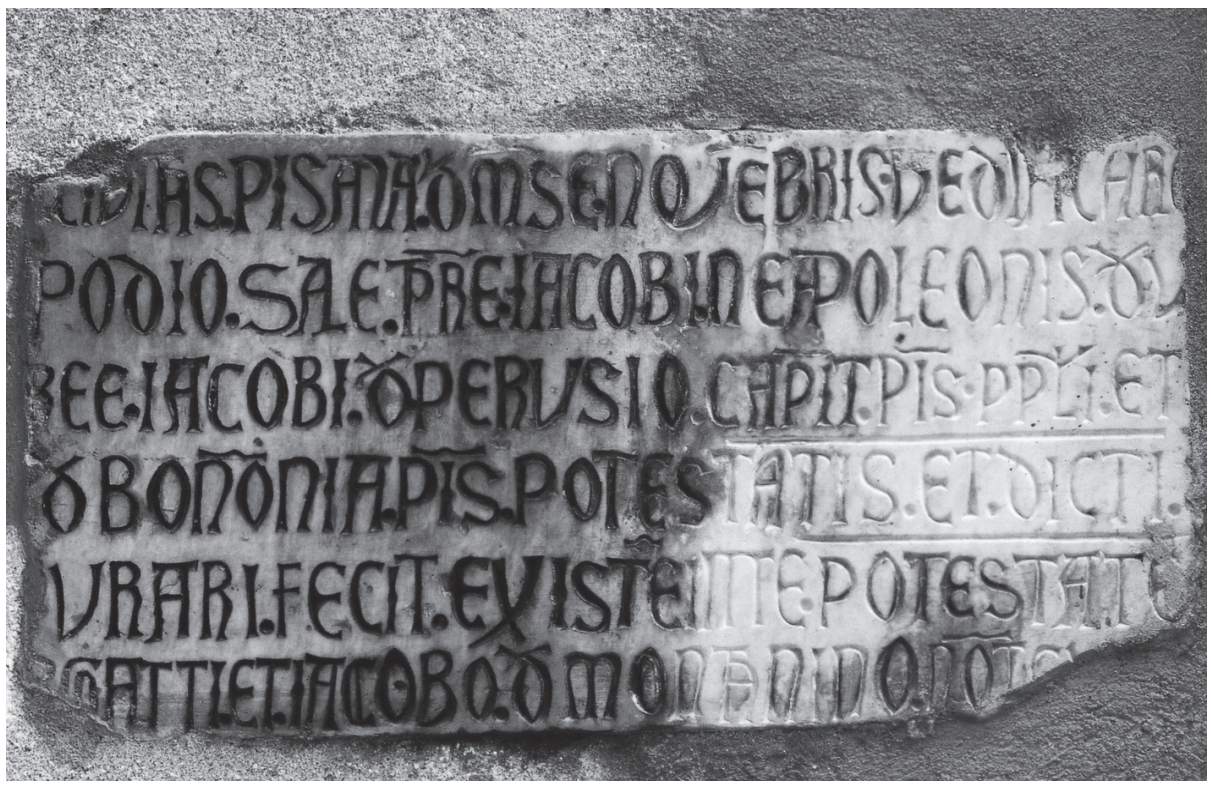

Abb. 3: Genua, Palazzo di San Giorgio, Innenhof, Pisanische Bauinschrift (Foto: Verfasserin).

mächtigkeit kommunalen Inschriften zukam, denn nur ihr Potential, den Gegner zu repräsentieren, ermöglicht es ihnen, auf seine Niederlage zu verweisen. ${ }^{22}$ Die Genuesen ließen es sich nicht nehmen, die Niederlage der Pisaner auch vor Ort in Lerici mit einer Inschrift präsent zu halten, in der Lerici selbst spricht: Genua „[...] lässt den weinen, der mich vernachlässigte, als er mich besaß“ - eine Reaktion auf die Pisaner Bauinschrift, deren ursprünglichen Platz sie möglicherweise jetzt einnahm. ${ }^{23}$

Im Gegensatz $\mathrm{zu}$ allen anderen hier diskutierten Monumenten besaß die in den Annalen zitierte Inschrift einen Text in Volgare. Damit ist ein Feld des „visibile parlare“ betreten, das hier nur angesprochen werden kann. Die Inschrift liegt uns in einer Brechung, in der Perspektive des (den Pisanern feindlich gesonnenen) Genueser Chronisten vor, möglicherweise ist sie Fiktion. Für beide denkbaren Ebenen, die der Überlieferung in den Annalen und jene einer von Pisanern formulierten Inschrift, ist zu fragen, was mit der Sprachebene des Volgare intendiert sein könnte: Sind die Sprecher oder die Leser damit charakterisiert? Soll eine mündliche Beschimpfung suggeriert werden, eingängig und wirkungsvoll durch den Reim? Dass VolgareInschriften nicht etwa grundsätzlich niedrige Register ziehen, sondern der Status der Auftraggeber oder die Qualität der Ausführung denen lateinischer Inschriften nicht

22 Für einen möglichen Vergleich, eine Inschrift der Mailänder Porta Romana, Müller 2002, 82.

23 [...] SIC FACIET FLENDO / QUI ME NEGLEXIT HABENDO; Vecchi 1992, 301; vgl. Müller 2002, 81 Anm. 192 mit der Übers. 
nachstehen müssen, machen Untersuchungen etwa der Bologneser Volgare-Inschriften deutlich. ${ }^{24}$ Für Florenz und Siena führt Maria Monica Donato zahlreiche Inschriften in Volgare im Kontext primär politisch motivierter Bilder auf, darunter metrische Schandinschriften, die den engsten Vergleich zu der den Pisanern zugeschriebenen Inschrift bilden. ${ }^{25}$ Wenn die Genueser Historiographie das Medium der Inschrift aufruft in einer Passage, in dem es um den zentralen Diskurs der kommunalen Ehre geht (die erst verletzt und dann wieder hergestellt wird), so läßt sich hier nachvollziehen, was Christoph Friedrich Weber für die Wappen und ihrem Stellenwert für die städtischen Chronisten des Trecento herausarbeiten konnte: diese

griffen [...] als Geschichtsschreiber [...] die Wappen, ihre Medien und ihre speziellen kommunikativen Kontexte auf, um sie im Interesse ihrer Dartellungsabsichten mit narrativen Strukturen zu verbinden.

Anhand des Mediums Inschrift können die Annalen bestimmte Praktiken und Handlungsmuster vorführen, und dabei ist es unerheblich, ob es genau diese Inschrift je gegeben hat.

Zwei weitere Genueser Inschriften des späten 13. Jahrhunderts sind eng mit Artefakten verbunden. 1290 hatten die Genuesen unter Corrado Doria für kurze Zeit die Hafenanlagen der Pisaner erobert und teilweise zerstört. Dieser Sieg war weniger bedeutend als jener sechs Jahre zuvor vor der Insel Meloria, aber die Erinnerung an ihn wurde zumindest in visueller Form besonders intensiv präsent gehalten. ${ }^{26}$ Den Genuesen gelang es, die Hafenkette zu durchbrechen und einen langen Strang davon abzutransportieren. In Genua wurden die Ketten zersägt und in Portionen aufgetrennt, die man an zahlreichen Kirchen, den Stadttoren und dem Kommunalpalast aufhängte (Abb. 4). ${ }^{27}$

Diese Trophäe stellte tatsächlich jedem, der sich in der Stadt bewegte, vor Augen, dass die Genuesen bis in das militär- und handelsstrategische Herz Pisas gelangt waren und suggerierte, dass der Hafen nunmehr schutzlos war. Es war vor allem ihre Omnipräsenz im Stadtbild, die dazu führte, dass die Hafenketten seit dem 15. Jahrhundert immer wieder in Stadtbeschreibungen und im Städtelob genannt wurden,

24 Breveglieri 1997.

25 Donato 1997; Vecchi 1992, 298 nennt für die Lunigiana als zwei Beispiele „delle poche in volgare“ zwei Inschriften erst aus der ersten Hälfte des 14. Jahrhunderts.

26 Hinweise, dass sogar dem Schmied, der die Ketten zu brechen vermochte, aus diesem Grund eine besondere liturgische memoria zuteil wurde, datieren offenbar erst aus der Neuzeit, vgl. Müller 2002, 94. Vgl. für die Hintergründe der Auseinandersetzungen mit Pisa im 12. und 13. Jahrhundert und ihre Rezeption den Überblick bei Polonio 2003, 161-212 und speziell die Beiträge in Mazzanti 1984 und Genova, Pisa e il mediterraneo 1984.

27 Ausführlich, auch zu der außergenuesischen Überlieferung, Müller 2002, 91-101, 220-222. Vgl. zu diesem Themenkomplex außerdem die Überlegungen bei Kedar 2012. 


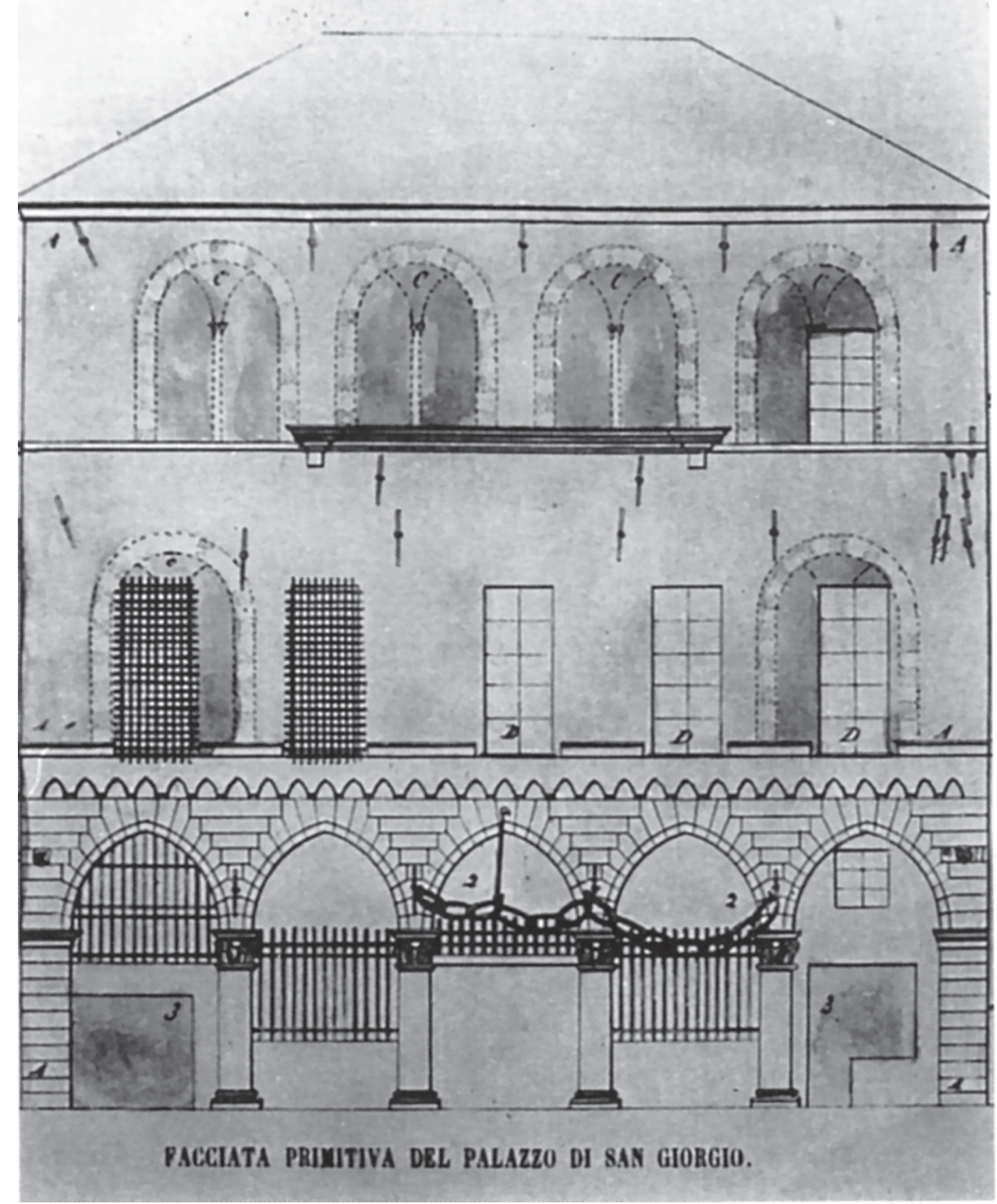

Abb. 4: Rekonstruktion der Fassade des Palazzo di San Giorgio (nach Carlo Cuneo, Memorie sopra l'antico Debito Pubblico. Mutui, Compere e Banca di S. Giorgio in Genova, Genua 1842, Tav. I). 
etwa von dem Diplomaten Giannozzo Manetti, der zu Genua in den Jahren 1435/36 hervorhob, dass die Ketten an den besonders belebten Orten der Stadt ausgestellt waren und jeder sie sehen musste, ob er wollte oder nicht. ${ }^{28}$ Als im Wortsinn massiv präsentes und vorgezeigtes Ding regte die Hafenkette eine Produktion von Bildern und abermals Inschriften an. Zwei Reliefs sind überliefert, die aus dem Anlass der Erbeutung der Ketten entstanden oder zumindest mit ihr in Zusammenhang stehen. Während das eine zu einem Museumsobjekt wurde (Abb. 5, 6), ist das andere, immer noch begleitet von zwei Gliedern der Hafenkette, weiterhin öffentlich und möglicherweise am ursprünglichen Ort ausgestellt, ein Umstand, der wiederum eine moderne Inschrift generierte (Abb. 7, 8). ${ }^{29}$ Beide Reliefs sind selbst Träger von Inschriften, Text und Bild nehmen jedoch in ganz unterschiedlicher Weise aufeinander Bezug.

Das einen knappen Meter breite Marmorrelief im Museo di Sant’Agostino (Abb. 5) stammt von einem Haus nahe der Porta Soprana, das selbst nicht mittelalterlichen Datums war, so dass über die ursprüngliche Anbringung nichts bekannt ist. ${ }^{30}$ Die in gotischer Majuskel ausgeführte Inschrift nennt das Datum September 1290 und führt aus, dass der „capitano del comune“ Corrado Doria Portopisano zerstört hat. Es folgt der Name des Auftraggebers: „Nic(olaus) d(e/a)“ und ein unleserlicher Namensteil. ${ }^{31}$

28 [...] Ianuenses igitur hac singulari adversus Pisanos victoria potiti [...] omnes Portus Pisani turres et arces insuper et Liburnam devastantes, omnia incendio et direptione fedarunt ; postremo ferream portus sui catenam in minutas quasdam partes perfregerunt quas, Ianuam delatas, celebrioribus urbis sue locis precipue et in hostio proprii portus et in publicis ianuis altius suspensas, ut quicunque vel ex mari vel ex terra urbem ingrederentur inviti etiam conspicerent, in memoriam profligatorum hostium infixerunt, Giannozzo Manetti 1974, 109 f.

29 Ob es sich bei den nicht im Zuge der italienischen Einigung zurückgegebenen Kettengliedern in Moneglia und Murta tatsächlich um die 1290 geraubten Ketten handelt, wurde materialtechnologisch m. W. bislang nicht untersucht. Spätere Ergänzungen, Ersetzungen und Kopien möchte ich nicht ausschließen, da auch später Hafenketten als Trophäen nach Genua gelangten und im 19. Jahrhundert die Rückgabe vieldiskutiert und umstritten blieb, vgl. Müller 2002, 221 f.; hier 94-101 zum Folgenden über die beiden Reliefs.

30 85x38x14 cm. Es ist unklar, worauf (abgesehen von einer Äußerung Federico Alizeris) in der jüngsten Publikation zu dem Relief die Aussage basiert, es ,proviene dal parato murario di un palazzo duecentesco della zona di Ponticello di proprietà del committente, che partecipò all'impresa in prima persona“, vgl. Pessa 2016, 221f. (Francesca Girelli). Croce 2008 diskutiert die baulichen Veränderungen im Vico Dritto di Ponticello; ebd., 42/43 oben (Zeichnung Guido Zibordi Marchesi) ist das Hafenrelief an dem Haus Vico Dritto di Ponticello, Ecke Borgo dei Lanaioli gezeichnet (vgl. ebd., 37 [Zeichnung Guido Zibordi Marchesi] sowie 33 [Zeichnung Enzo Bifoli]). Treffen diese Rekonstruktionen zu (die Rekonstruktion „fine 1400“ zeigt das Hafenrelief nicht, das Werk wird im Text jedoch nicht diskutiert), dann widerspricht bereits die Einmauerung in ca. 4 m Höhe der Annahme, es handele sich um den ursprünglichen Anbringungsort.

31 Origone/Varaldo 1983, 44f. Nr. 10 transkribieren den Text wie folgt (abweichend hier in Großschreibung): MCC ${ }^{\circ}$ XXXXX ME(N)SE SEPT(EM)B(RIS). / D(OMI)N(U)S CU(N)R(ADUS) AUR(IE) CAP(I) T(ANEUS) CO(MMUNI)I $<$ S > ET P(O)P(U)LI IAN(UENSIS) / DESTRU / SIT POR / TU(M) PISANU(M). NIC(OLAUS) D(E) G[UIEL]MO FECIT FIERI HOC. 


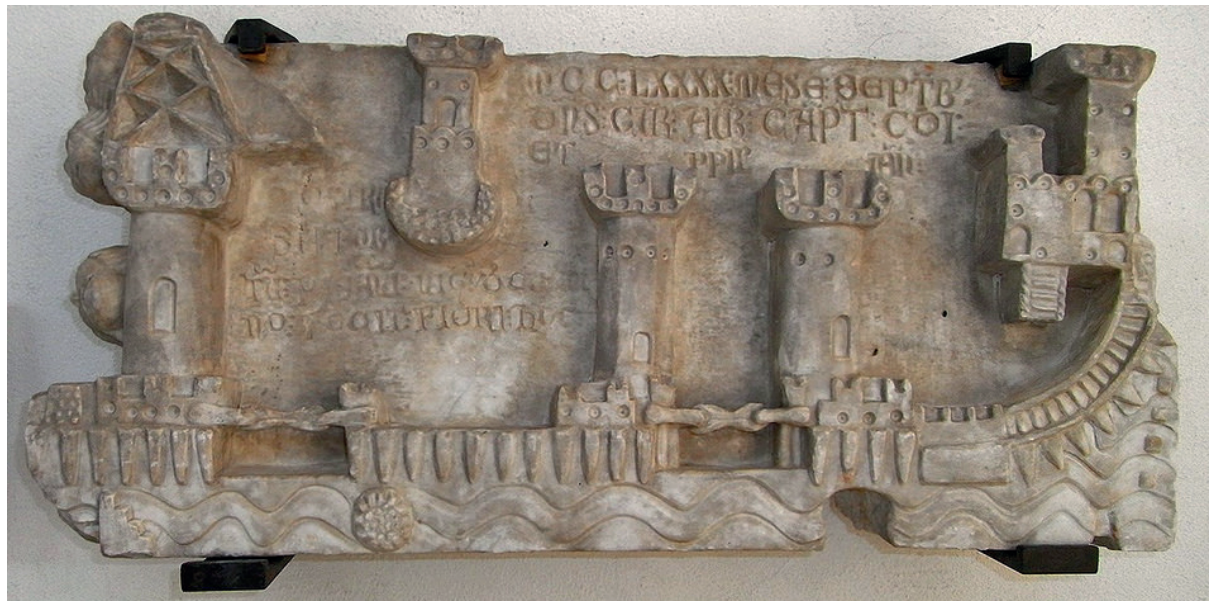

Abb. 5: Relief mit der Darstellung von Portopisano (mit freundlicher Erlaubnis des Museo di Sant'Agostino, Genua).

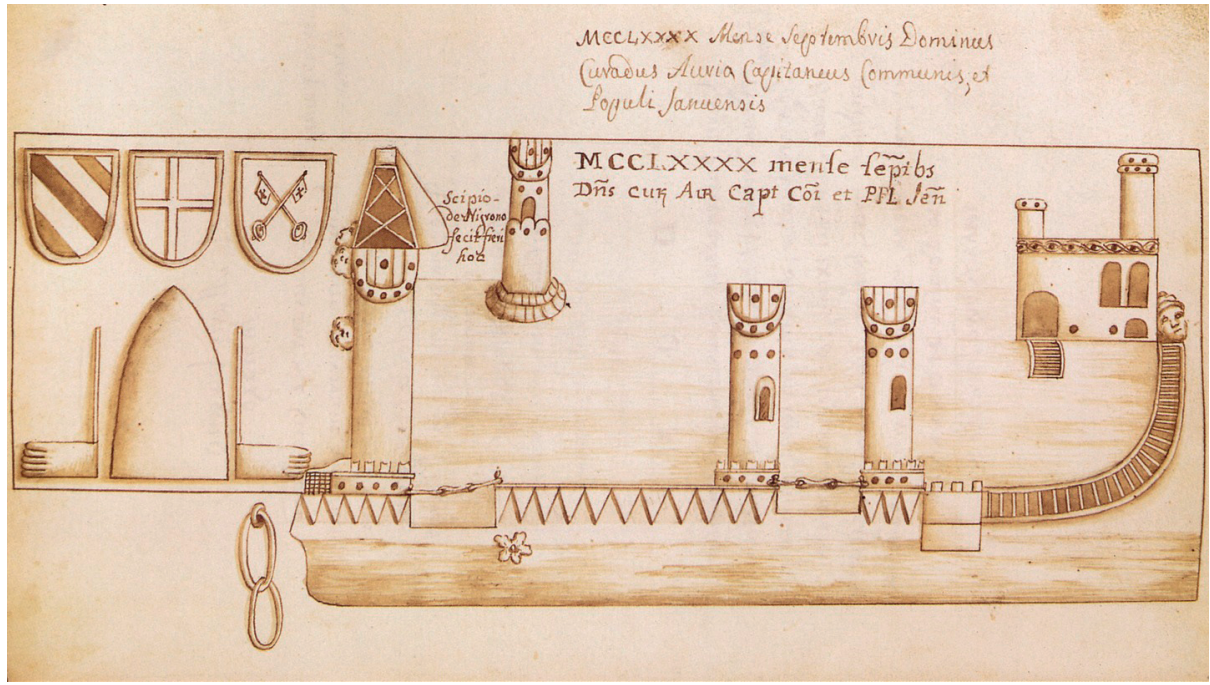

Abb. 6: Domenico Piaggio, Nachzeichnung des Reliefs mit der Wiedergabe von Portopisano in den Epitaphia, sepulcra et inscriptiones cum stemmatibus, marmorea et lapidea, existentia in ecclesiis genuensibus, MS1720, Genua, Biblioteca Civica Berio, Biblioteca Berio, Bd. 5 (m.r.V.4.5), fol. 285r (alte Numerierung 283). 


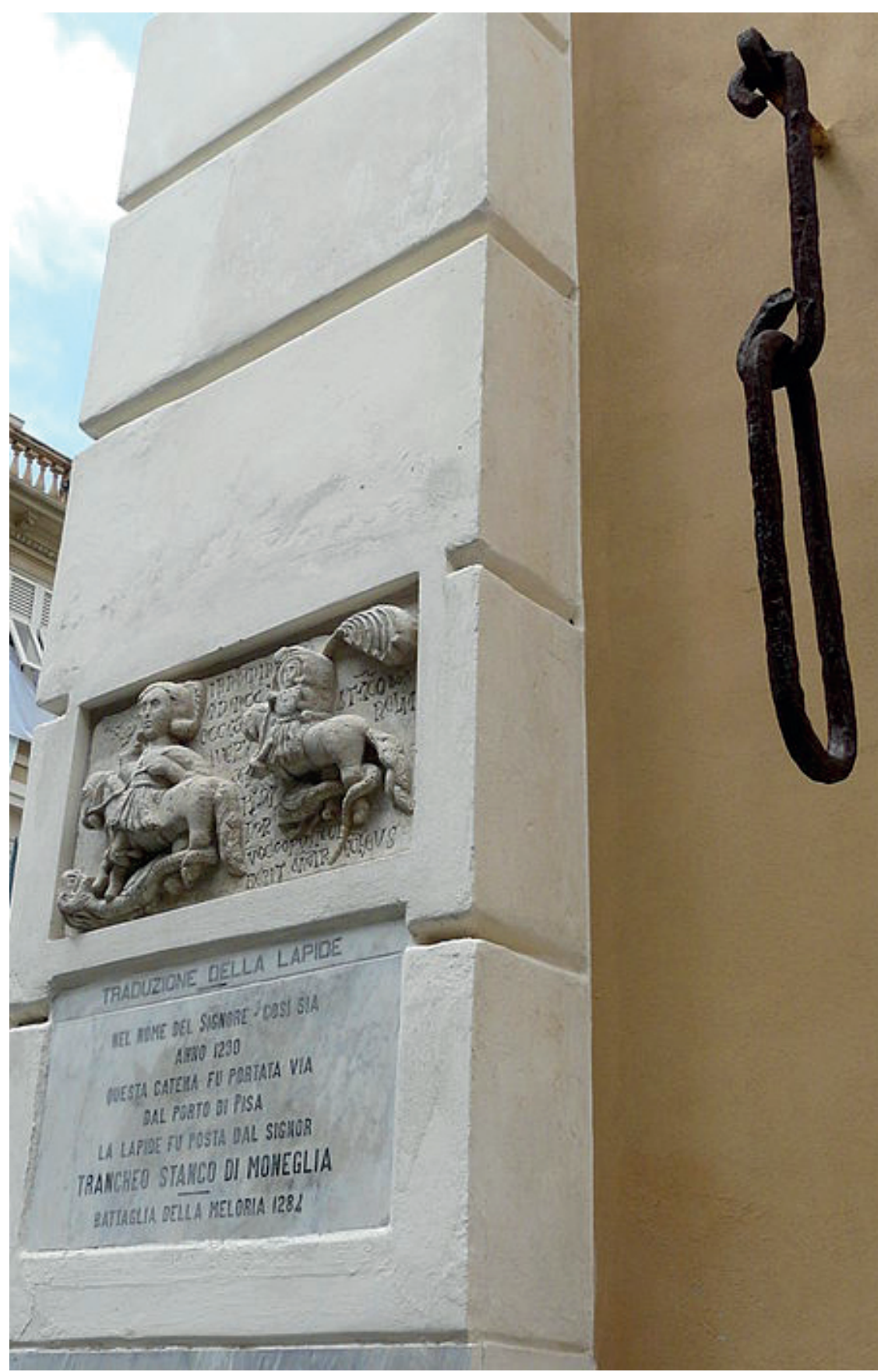

Abb. 7: Moneglia, Santa Croce, Südwestecke mit Relief, Gliedern der Pisaner Hafenketten und moderner Inschrift (Davide Papalini, CC BY-SA 3.0, via Wikimedia Commons, https://commons. wikimedia.org/wiki/File\%3AMoneglia-chiesa_santa_croce-catena.JPG). 


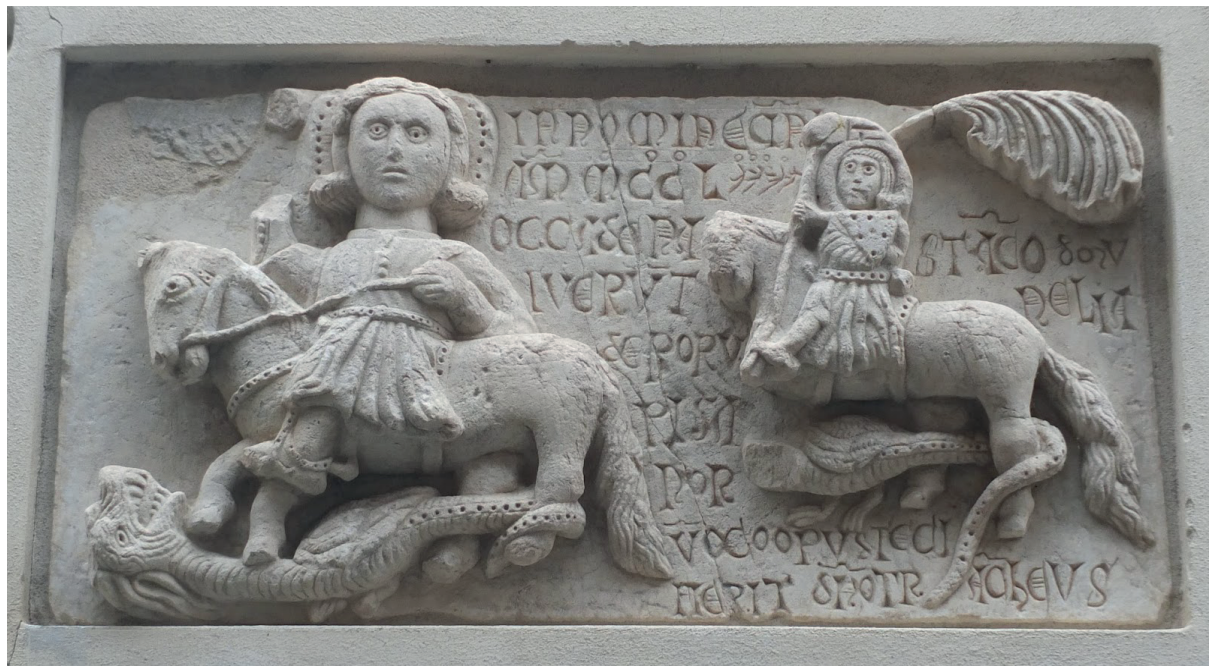

Abb. 8: Moneglia, Santa Croce, Relief mit Drachenreitern und Inschrift zur Einnahme des Pisaner Hafens. Mit freundlicher Erlaubnis von Martha und Mike Mazzaschi (www.apathtolunch.com).

Die übliche Ergänzung „de Guielmo“ ist nicht zwingend, da statt G(...)MO auch C(...)NO eine mögliche Lesung ist. Überraschenderweise blieb sogar in der älteren Lokalforschung die Zeichnung unberücksichtigt, mit der Domenico Piaggio um 1720 das Relief mit heute fehlenden Wappen aufnahm (Abb. 6). Da es sich bei dem Wappen heraldisch rechts des Genueser Kreuzwappens um jenes der Fieschi handeln könnte, ${ }^{32}$ ist ein Bezug zu Cogorno möglich, wo die Familie im Herzen des ursprünglichen Territorialbesitzes mit San Salvatore dei Fieschi im 13. Jahrhundert aufwendige Stiftungen realisiert hatte. ${ }^{33}$ Die Präsenz des kommunalen Wappens widerspricht in jedem Fall dem mehrfach angenommenen ,privaten“ Charakter von Relief und Inschrift. ${ }^{34}$

Den Hafen charakterisieren drei untereinander durch Ketten verbundene Türme, deren rechter über eine Landzunge mit einem palastartigen Bau verbunden ist. Auf einer Insel im Hintergrund erhebt sich ein einzelner Turm. Vor der Hafensperre sind eine weitere Insel und eine treppenförmige Anlage gezeigt. Schematische Wellenlinien deuten Wellen und Seegang an. Aus dem größten Turm links, den eine Laterne

32 Den Hinweis darauf verdanke ich Andreas Rehberg. Zu dem Wappen Genuas Weber 2011, 133-135. Es ist verlockend, das Schlüsselwappen im Zusammenhang mit dem Fieschiwappen als päpstliches Wappen zu deuten, zumal im Kontext eines möglichen Bezugs auf Cogorno (s. u.), aber Hadrian V. Ottobuono Fieschi war bereits 1276 verstorben und es ist unklar, wer aus Cogorno in den Jahren danach das Wappen mit den gekreuzten Schlüsseln noch hätte führen dürfen.

33 Ein Nicolò di Cogorno ist in dem Testament des Bertolino Fieschi aus dem Jahr 1313 erwähnt, vgl. Firpo 2015, 23 Anm. 23.

34 So etwa bei Dagnino 1992a, 199 („Epigrafe e rilievo [...] non si collocano nei parametri di un messaggio pubblico"). 
auszeichnet, blicken drei Köpfe heraus, ein weiteres Gesicht wird neben dem Gebäude rechts erkennbar. Die Hafenanlagen von Pisa sind heute verlandet, Grabungen und literarische Quellen legen aber nahe, dass sich das Relief an der realen Topographie orientiert, und Gabriella Rossetti konnte auf dieser Grundlage die Türme benennen. ${ }^{35}$

Die einem stilkritischen Ansatz kaum zugänglichen formalen Charakteristika des Reliefs, etwa die schematische Figurendarstellung, die unregelmäßigen Buchstaben und die unbefriedigende Schriftverteilung sollten nicht den Blick dafür verstellen, dass es sich um ein in der Gattungstypologie wie der Programmatik bemerkenswertes Erinnerungsmal handelt. Als monumentale mittelalterliche Hafendarstellung bleibt das Relief offenbar ein Unikum. Indem Seitenansicht und Aufsicht nebeneinanderstehen und der Bildhauer sich auf die Großbauten konzentriert, sind Charakteristika von gemalten Stadtabbreviaturen aufgenommen, denen hier durch die Umsetzung in Marmor Monumentalität und Dauerhaftigkeit verliehen wird. Diese Monumentensetzung lässt finanziellen Aufwand erkennen und impliziert den Anspruch einer öffentlichkeitswirksamen Ansprache, ohne dass sich ausmachen lässt, inwiefern der Auftraggeber mit dem Kriegsereignis, mit dem als verantwortliche Einzelperson so hervorgehobenen Doria, oder mit der Kommune verbunden war, deren Wappen er verwendet. Im Verhältnis zu der Inschrift erfüllt das Relief die Funktion, bildlich vor Augen zu stellen, was die Zerstörung der Hafenanlagen eigentlich so bedeutsam macht. Nicht die Schlacht selbst oder ihr Ergebnis sind gezeigt, also dass, was der Inhalt der Inschrift ist, sondern der bemannte und befestigte Hafen vor der Zerstörung. Die Sperrketten sind durch ihre auf wenige Glieder verkürzte, bedeutungsperspektivisch vergrößerte Wiedergabe und durch die mit Blick auf die tatsächliche Nutzung unrealistische, da über der Wasseroberfläche gespannte Anbringung besonders hervorgehoben, ebenso die Besatzung der Türme. Es wird damit betont, wie gut gesichert der Hafen war, wodurch die Leistung des Doria um so größer erscheinen muss. Das Bild allein wäre ohne die Inschrift in seiner Intention aber unverständlich, und insofern wird hier das Potential beider Medien verbunden. Allerdings dürften die starken Abkürzungen, die sich bei einer geschickteren Aufteilung der gravierbaren Fläche hätten vermeiden lassen, die Lesbarkeit eingeschränkt haben. Als drittes Element kommen die als dinglicher Beleg ursprünglich daneben aufgehängten realen Kettenglieder hinzu: Das, was wie im Relief verbildlicht zuvor den pisanischen Hafen sicherte, hat der Doria der eigenen Stadt einverleibt und den Gegner damit entblößt. Diese Strategie, Sieghaftigkeit zu demonstrieren, indem man das Überwundene präsentiert und nicht den Akt der Überwindung, greift die antike Praxis auf, im Triumphzug nicht nur Beute, sondern auch gemalte Darstellungen der eroberten Städte mit-

35 Rossetti 1989, 269, 271 (von links Torre del Fanale mit Laterne, Torre Soarsa, Torre di San Vito, daneben die domus magna, von der eine Brücke zur Torre di San Vito verlief, hinten die Torre Magnale). 
zuführen, ein Brauch, dessen Kenntnis etwa über die Lektüre von Plinius und Ovid vermittelt worden sein könnte. ${ }^{36}$

Das rund $75 \mathrm{~cm}$ breite Relief in Moneglia (Abb. 8) ist in seinem Bild-Text-Verhältnis schwieriger $\mathrm{zu}$ beurteilen, da die Ikonographie nicht eindeutig ist. Die in fehlerhaftem Latein ausgeführte und wegen der Abkürzungen und der unregelmäßigen, zwischen Majuskeln und vereinzelten Minuskeln schwankenden Schrift heute wie wohl auch im 13. Jahrhundert schwer lesbare Inschrift kann wie folgt übersetzt werden: „Im Namen des Herren Amen. 1290 raubten sie diese Kette aus dem Hafen der Pisaner. Dieses Werk ließ Herr Trancheus Stanco aus Moneglia machen.“37

Die lokale Adelsfamilie der Stanco da Moneglia ist mehrfach in Notariatsakten erwähnt, ein Trancheo ist bislang nicht auszumachen. Vermutlich hatte er selbst an der Hafeneinnahme teilgenommen und die Trophäe für sich beanspruchen können. Ein Problem stellt die Benennung der Reiter dar: Sie wurden als Teilnehmer der Schlacht, ja als Corrado Doria selbst identifiziert, zuletzt sah man in dem linken Reiter Trancheo Stanco und in dem rechten den hl. Georg, den Stadtpatron Genuas. ${ }^{38}$ Ein Adeliger hätte sich aber kaum in demselben Bildschema und deutlich größer als der Heilige darstellen lassen; der Drache wäre dann nur allegorisch zu verstehen. Es dürfte sich um zwei Ritterheilige handeln, da beide einen breiten Wulst hinter den Haaren als Heiligenschein um den Kopf geführt zeigen. Als zweiter Drachenbezwinger neben dem hl. Georg kommt Demetrius von Saloniki in Betracht, der im Westen als Schlachtenhelfer verehrt, allerdings selten dargestellt wurde. Die Figuren bleiben damit ohne erkennbaren direkten Bezug auf das in der Inschrift erwähnte Ereignis; gezeigt sind wohl diejenigen, denen der Auftraggeber für den Sieg und die gesunde Heimkehr dankt. Die Reiter bleiben ebenfalls ohne visuellen Bezug zur Trophäe, die in das Monument jedoch durch die Inschrift „hoc cadena“ eingeschlossen wird. Weniger der Ruhm der Genuesen oder ihres Anführers als vielmehr die Anempfehlung eines Kriegsteilnehmers an zwei Heilige scheint hier das Thema zu sein, denn auch in der Inschrift wird das Geschehen auf die Überführung der Beute reduziert. Ein kommunales Ereignis ist damit Anlass einer von einer Einzelperson initiierten, in der Gemeinschaft wirksamen Monumentensetzung, wobei die in kommunalen Erin-

36 Plinius, nat. V, 5, 36 (et hoc mirum, supra dicta oppida ab eo capta auctores nostros prodidisse, ipsum in triumpho praeter Cidamum et Garamam omnium aliarum gentium urbiumque nomina ac simulacra duxisse, zu Cornelius Balbus); Ovid, ars I, 219f. (Atque aliqua ex illis cum regum nomina quaeret, Quae loca, qui montes, quaeve ferantur aquae, Schilderung eines Triumphzugs); siehe auch Claudian, Stil. III, 24 (hi famulos traherent reges; hi facta metallo oppida vel montes captivaque flumina ferrent, Schilderung eines Triumphzugs). Wer diese Lektüre wiederum konkret an den Auftraggeber vermittelt haben könnte, lässt sich nicht fassen.

37 IN NOMINE D(OMI)NI / AM(EN) MCCLXXXX / OC CADENA / TUERU(N)T / DE PORTU / PISA / NOR /U(M) OC OOPUS FECI / FIERIT D(OMI)NO TRA(N)CHEUS / STA(N)CO D(E) MU/NELIA, vgl. Remondini 1878, 36.

38 Für das Relief vgl. Müller 2002, 99 f.; Di Fabio 2007, 295 f. (mit der Identifikation der Reiter als Trancheo und hl. Georg). 
nerungsmalen verwendeten Medien von Bild, Inschrift und Trophäe übernommen sind. Vermutlich sind die Bestandteile dieses Monumentes nicht gleichzeitig entstanden: die Inschrift wurde offenbar erst angebracht, als der rechte Reiter bereits bestoßen war, denn die heute fehlende Vorderhand des Pferdes und der Kopf des Drachens hätten die Buchstaben verdeckt. ${ }^{39}$ Dies erklärt jedoch nicht, warum das Layout der Inschrift einem Lese-Verständnis geradezu entgegensteht. Bei der Schriftform fallen die Auflösung der Balken einiger Buchstaben in drei feine Striche und die Ornamentalisierung des „X“ ins Auge, wofür es offenbar in dem umfangreichen Corpus der Genueser Inschriften keine Vergleiche gibt. Beides bezeugt einen bewussten Schriftakt, bei dem das Textverständnis nicht das primäre Ziel war.

Die agency des Objekts blieb den Hafenketten auch in der Moderne eigen, beziehungsweise wurde ihnen im Risorgimento wieder neu zu eigen. Die Kommune beschloss, sie im Zuge der italienischen Einigung an Pisa zurückgegeben, ein Akt, der jedoch viel diskutiert und - wie die Kettenglieder in Moneglia bezeugen - nie ganz vollzogen wurde. ${ }^{40}$

Das unter dem Aspekt der Indienstnahme kommunaler Medien durch Einzelpersonen beziehungsweise Familien aufwendigste Genueser Ensemble stellen die Fassaden des Palastes von Lamba Doria (Abb. 9, 10) und der Familienkirche der Doria, San Matteo (Abb. 11, 12, 13), dar. Möchte man allein die fünf mittelalterlichen Inschriften lesen, die hier als Ehreninschriften für jeweils einen Doria aus Anlass seines Schlachtensieges eingemeißelt wurden, dann sind nicht weniger als 140 Meter Inschriften abzuschreiten und zu entziffern (die Rückwege zum Zeilenanfang nicht mitgerechnet!). ${ }^{41}$ Auch diese Inschriften beziehen sich bemerkenswert häufig auf Objekte (ohne dass es sich etwa um Bauinschriften o.ä. handelt).

Bereits die in der Ausführung vermutlich früheste Inschrift, jene für Lamba Doria an dessen Palast, nennt gleich zu Beginn einen Gegenstand. ${ }^{42}$ Bei der Seeschlacht gegen die Venezianer vor der Insel Korçula im Jahr 1298 erbeutete Lamba als Admiral nicht nur 18 Schiffe samt der gefangenen Besatzung, sondern auch einen „Engel“ angelus captus fuit, der, worauf das iste davor hindeutet, in nächster Nähe angebracht war. ${ }^{43}$ Was genau mit angelus bezeichnet war, ist intensiv diskutiert worden, mit dem Ergebnis, dass es sich entweder um den antiken Sarkophag handeln könnte, der

39 Für diese Beobachtung danke ich Rahel Meier.

40 Vgl. etwa Costa 1859/1892. Der Consiglio Comunale di Genova entschied positiv über die Rückgabe. $41 \mathrm{Zu}$ den Inschriften siehe Giovè Marchioli 1994, bes. 276f.; Müller 2002, 126-133, hier auch zum Problem der Datierung und der Reihenfolge, in denen die Inschriften angebracht wurden; 116-122 zu dem im folgenden erwähnten Sarkophag und seinen möglichen Bezug zu der Inschrift am Palast des Lamba Doria.

42 Die Inschriften zu den Doria sind transkribiert bei Silva 1987, 72 f., 78, 81 und, mit geringen Korrekturen, bei Müller 2002, 243-245.

431298 [...] DIE VII SE(P)TEMBRIS ISTE ANGELUS CAPTUS FUIT IN GULFO VENECIARUM IN CIVITATE INSULE SCURZOLE ET IBIDEM FUIT PRELIUM [...]: 


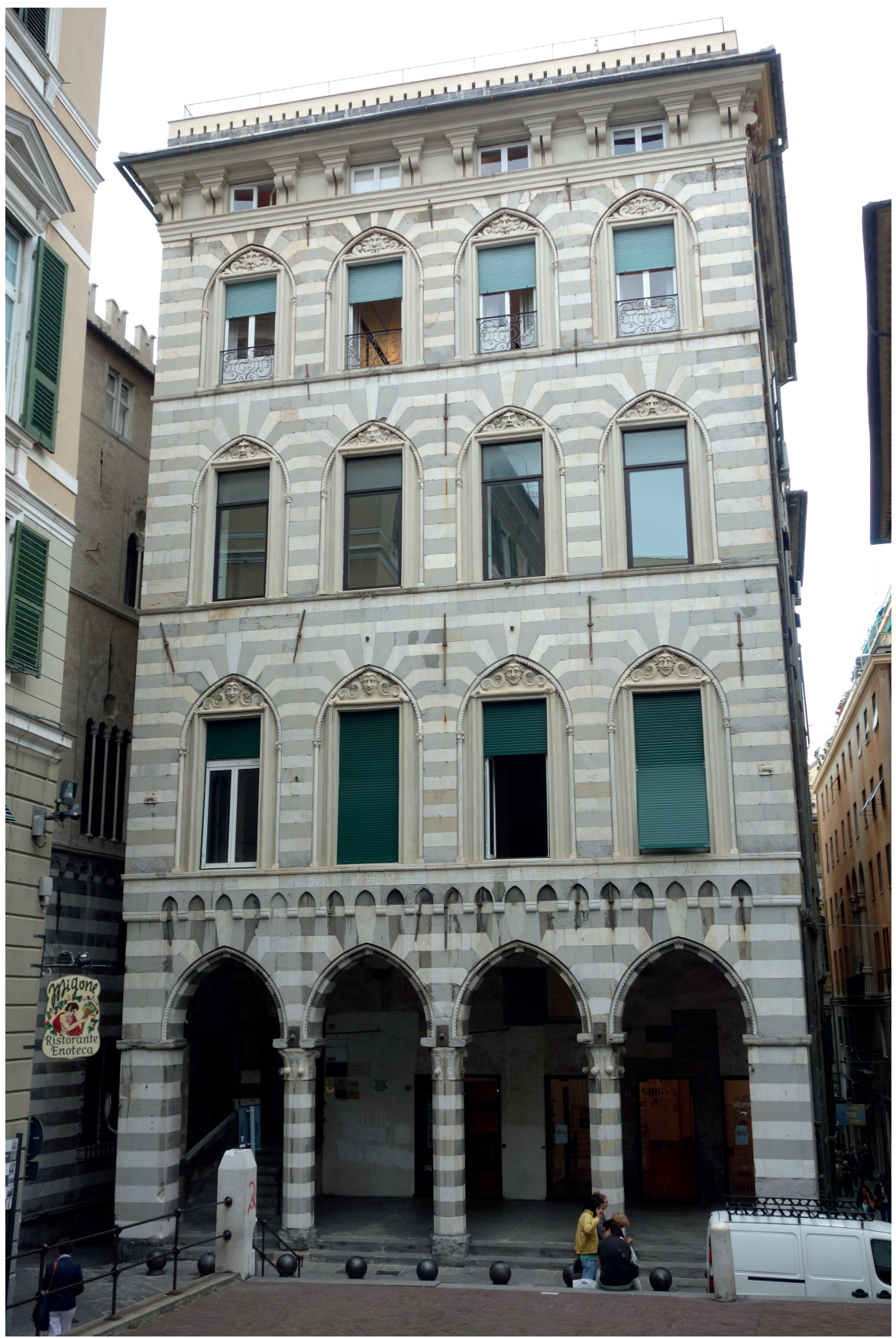

Abb. 9: Genua, Palast des Lamba Doria (Daderot, Creative Commons CCO 1.0 Universal Public Domain Dedication, via Wikimedia Commons, https://commons.wikimedia.org/wiki/File:Palazzo_ Doria_Lamba_-_Genoa,_Italy_-_DSC01947.JPG). 


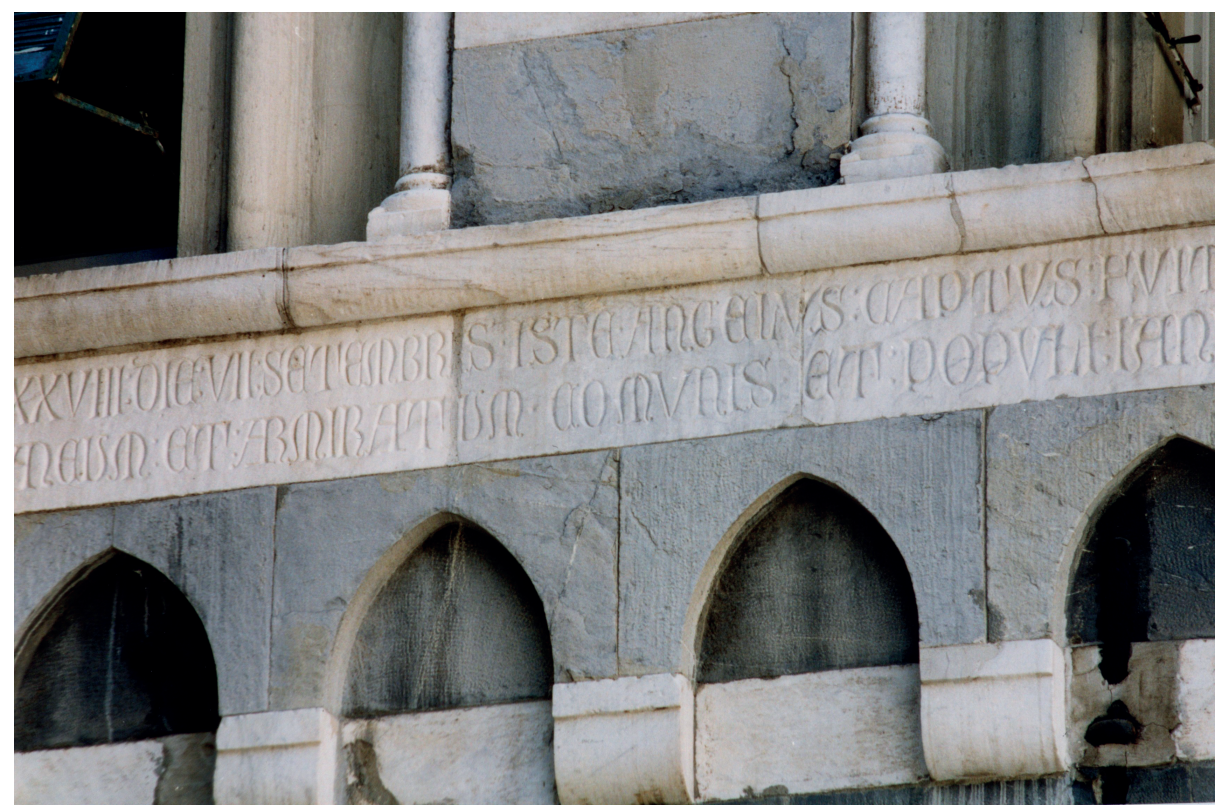

Abb. 10: Genua, Palast des Lamba Doria, Detail der Inschrift zu Lambas Sieg 1298 (Foto: Verfasserin).

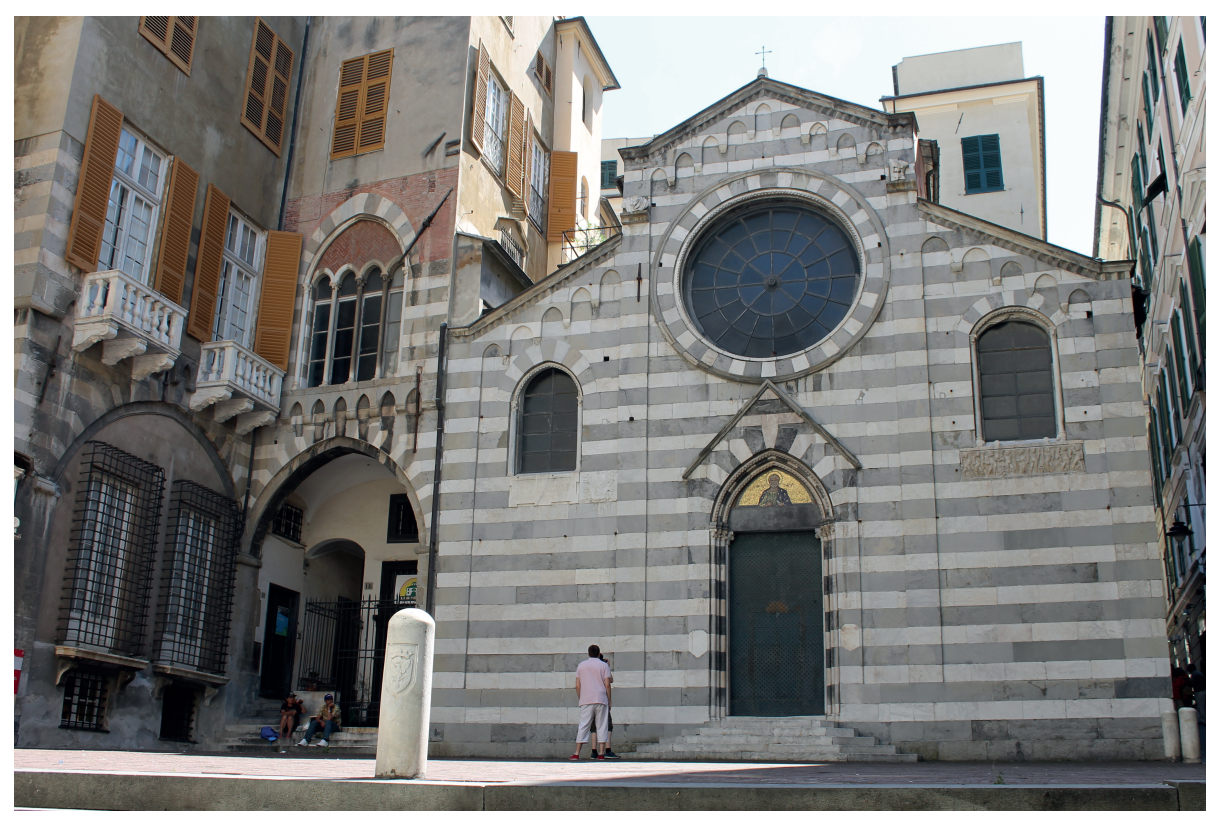

Abb. 11: Genua, San Matteo (Foto: Verfasserin). 


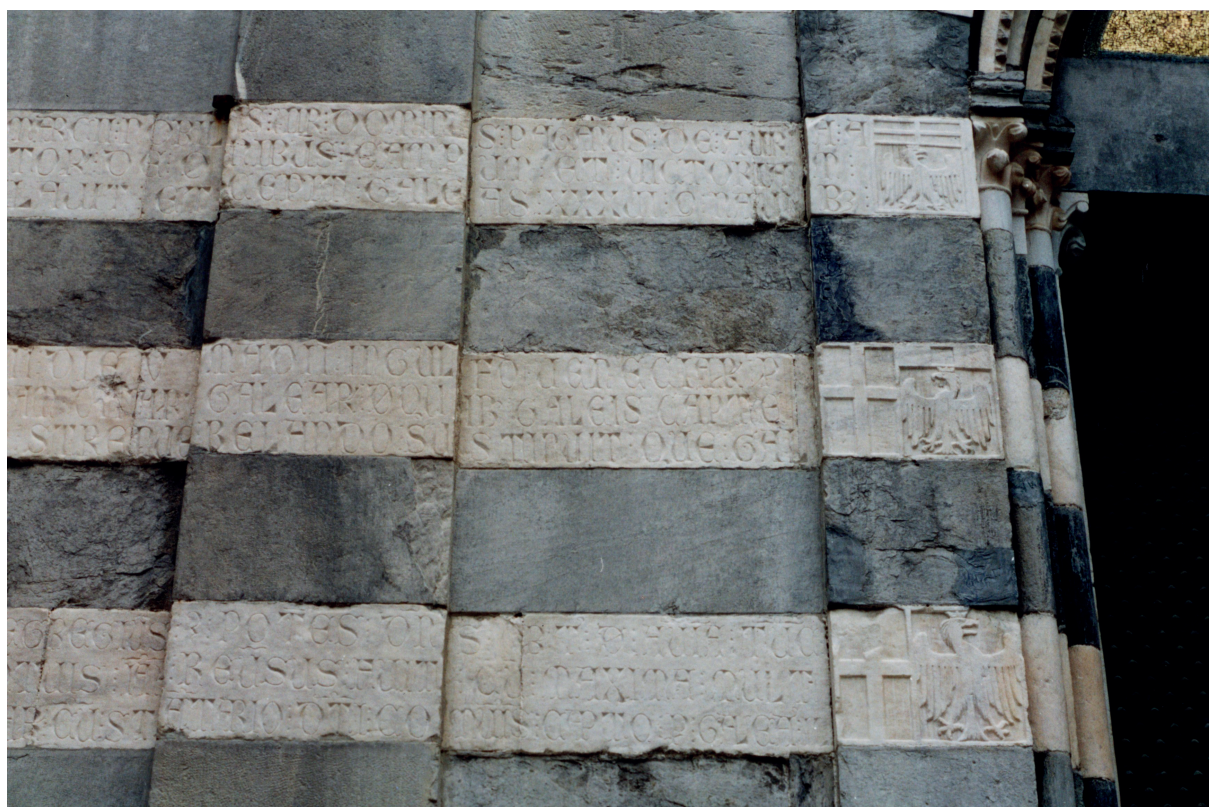

Abb. 12: Genua, San Matteo, Detail mit Wappen und den Inschriften, die die Siege des Pagano, des Luciano und des Oberto Doria rühmen (1352 und 1354, 1379, 1284) (Foto: Verfasserin).

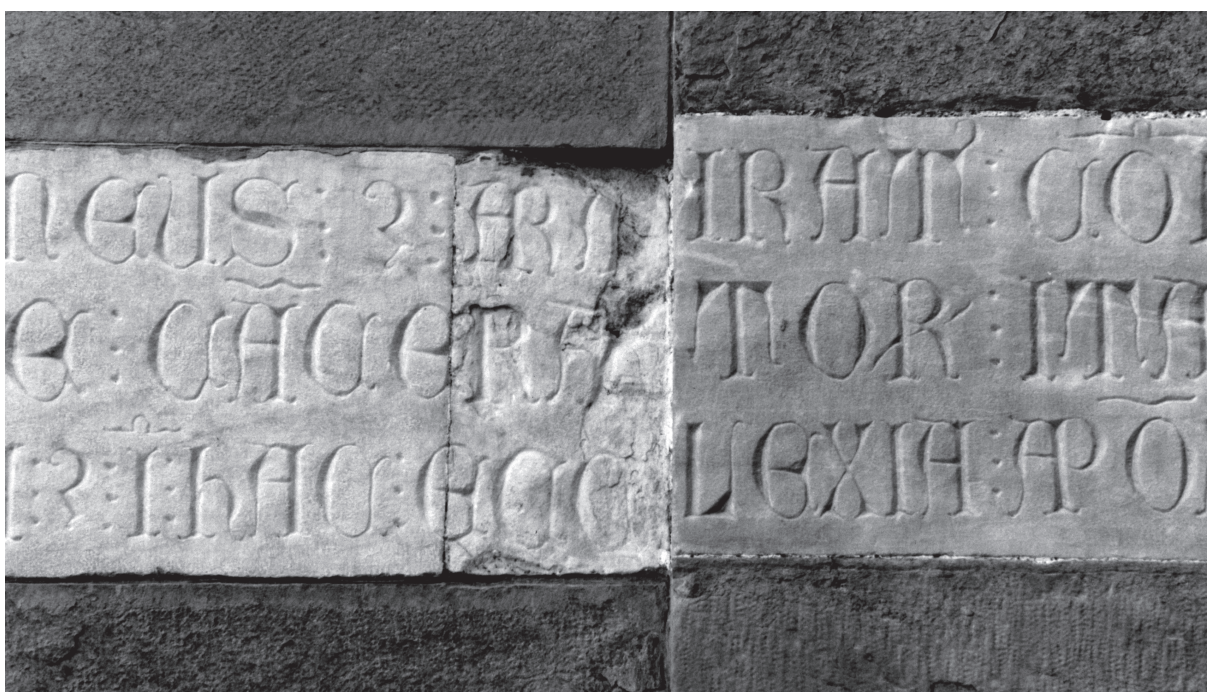

Abb. 13: Genua, San Matteo, Detail der Inschrift, die Oberto Dorias Sieg 1284 rühmt (Foto: Verfasserin). 
Lamba später als Begräbnisstätte diente und in Zweitverwendung in die Fassade von San Matteo einmauert wurde (und tatsächlich - allerdings mehrere - „Engel“ zeigt), oder um ein nicht näher bekanntes und heute verlorenes anderes Objekt.

Eine im Formular ganz ähnliche Inschrift für den damaligen Capitano del Popolo und Admiral Oberto Doria feiert den Sieg über die Pisaner vor der Insel Meloria 1284. Erwähnt werden nicht nur die Schiffe und die vielen adeligen Gefangenen, sondern auch die Standarte und das Siegel - gemeint sein dürfte das Siegeltypar - der Pisaner Kommune. Beide seien „in diese Kirche gebracht worden“. ${ }^{44}$ Wie das Siegel aufbewahrt wurde, ist nicht bekannt, es dürfte primär durch die Inschrift Präsenz gewonnen haben. Das Feldzeichen war wohl als Bekrönung der Fassade angebracht, denn neuzeitliche Darstellungen belegen mehrere Aufsätze auf dem Giebel und an der Traufe, ohne dass die Standarte aus der Meloriaschlacht eindeutig zu identifizieren wäre.

Die Entstehungsumstände der Inschrift an Lambas Palast erlauben es, das Verhältnis von Inschrift und Objekt an den Bauten der Doria zu präzisieren. In einer ausführlichen Passage zu der Schlacht vor Korçula erwähnt der Geschichtsschreiber, Notar und Dichter Ferreto de'Ferreti da Vicenza, dass es sich bei Palast und Inschrift um Ehrungen der Kommune gehandelt habe: ${ }^{45}$ Sie, die Amtsträger der Kommune, „beschlossen, dass für ihn [i. e. Lamba] zum ewigen Ruhm seines Namens auf öffentliche Kosten ein hoher Palast erbaut werde. Und sie gaben Anweisungen, nach Art der Vorfahren mit passenden Bildern und goldenen Buchstaben, eingraviert in Stein, Art und Grund des Krieges sowie die Anzahl der Gefangenen und der Schiffe aufzuschreiben. Und der Zahn der Zeit verhindert nicht, dass über eben dieser Vorhalle des Palastes (...) die ganze Begebenheit gelesen werden kann“. Diese für die Wirkung und Rezeption von Inschriften wichtige Quelle erweist, dass Palast und Inschrift als kommunales Ehrenmonument verstanden werden konnten, welches das Exemplum des Einzelnen allen vor Augen stellte. Damit ist ein antikes Konzept aufgegriffen, das nicht durch antike Inschriften vermittelt worden sein dürfte, die ein anderes Formular zeigen, sondern literarisch über Autoren wie Johannes von Salisbury, der

441284 [...] FUIT CAPT(US) ALB(ER)TUS / MOLEXIN(US) DE VENECIIS TU(N)C POTESTAS (ET) D(OMI)NU(S) GENERAL(IS) GUER(R)E CO(MUN)IS PISAR(UM) CU(M) STA(N)TARIO D(IC)TI COMU(N) IS CAPTO P(ER) CALEA(M) IL(L)OR(UM) D(E) AU(R)IA (ET) I(N) HA(N)C ECCLEXIA(M) APO(R)TATO CU(M) SIGILO D(IC)TI CO(MUN)IS [...]. Zur Schlacht vor Meloria siehe die Literatur in Anm. 26 sowie Del Punta 2015.

45 Ferreto schreibt in seiner Historia rerum in Italia gestarum um 1330: ne fama eius, ob tantam sibi partam victoriam, evo subeunte, immemorabilis extinguatur, illi atrium sublime marmore gispove contextum ad perpetuam sui nominis gloriam construi sumptibus publicis statuere, in quo priorum more ymaginibus propriis et litteris aureis, belli modum et causam, captivorumque et ratium numerum, ceso lapide, imprimi mandavere. nec obest adeo temporis lapsa vetustas, quin adhuc supra ipsum atrii vestibulum, nisi forte seditiones inter urbis illius corruerit, res tota legi possit; Ferreto de'Ferreti Vicentino 1908, 122. 
die rühmende und erinnernde Wirkung von Inschriften an römischen Triumphbögen beschreibt. ${ }^{46}$

Die Inschriften an der Kirchenfassade übernehmen das Formular der Ehreninschrift des Palastes sowie das Konzept, den Text auf einer Steinlage über die Fassade hinwegzuführen. An der Kirche werden sie von dem Wappen der Kommune und dem Adlerwappen der Doria begleitet, die ein Verständnis auch der Inschriften an der Kirche als kommunale Ehrungen suggerieren. Mit der Verbindung von Inschriften und Trophäen wird ebenfalls auf eine kommunale - und nicht nur Genueser - Praxis rekurriert. Die Verbindung visueller und textueller Strategien scheint hier wiederum darauf ausgelegt, eine breitere Ansprache zu erreichen und die Wahrheit des Gesagten zu untermauern. Jeder Betrachter dürfte von der Monumentalität und den ästhetischen Qualitäten der Inschriften überwältigt worden sein. Wer den Text las, der fand in den Trophäen den Beleg für seinen Inhalt; wer ihn nicht las oder nur eingeschränkt verstand, der konnte allein aufgrund der Präsenz der Inschriften und des kommunalen Wappens auf ihren offiziellen Charakter schließen - sie erhielten, wie es Nicoletta Giovè Marchioli für die kommunale Epigraphik formulierte:

[...] una funzione ipercomunicativa: essa [die Inschrift] assume [...] un valore iconico assoluto [...]; L'epigrafe dunque diventa un elemento quasi assolutamente materico, significativa solo per il fatto di essere stata scritta in un preciso momento [...] e di essere stata collocata in un preciso punto, indipendentemente dalla sua leggibilità. ${ }^{47}$

Für die Trophäen konnte der Betrachter bereits aus dem Ort, an dem man sie präsentierte, folgern, dass es sich um Beutestücke der Doria handelt.

Anstelle weiterer Trophäenmonumente ${ }^{48}$ soll ein ganz anders ausgerichtetes epigraphisches Ensemble den Beitrag beschließen, das in seiner Programmatik gerade in jüngster Zeit gut untersucht worden ist, dessen bildliche Bestandteile aber wenig Aufmerksamkeit erhielten. Die innenpolitische Entwicklung Genuas wurde im späten 13. Jahrhundert durch den Gegensatz guelfischer und ghibellinischer Adelsparteien geprägt. ${ }^{49}$ Innerstädtische Kämpfe führten $1296 \mathrm{zu}$ einem Großfeuer, bei dem die Kathedrale in Teilen ausbrannte. Die bis 1317 andauernde Neuausstattung, von der Kommune finanziert, wurde in zwei monumentalen Bauinschriften festgehalten. ${ }^{50}$

46 Vgl. Herklotz 1999.

47 Giovè Marchioli 1994, 283, 285. Ein anderes Monument, in dem die Doria das Wappen der Kommune beanspruchten und offenbar auch beanspruchen konnten, ist das Grabmal Pagano Dorias, der mit der Kreuzfahne in der Hand gezeigt ist, vgl. Weber 2011, $165 \mathrm{f}$.

$48 \mathrm{Zu}$ erinnern ist etwa an die beiden Reliefs mit venezianischen Markuslöwen, die 1380 in Pula bzw. Triest erbeutet worden waren. Die Hoheitszeichen wurden in Genua in San Marco al Molo bzw. am Palazzo Giustiniani eingemauert und mit einer erläuternden Inschrift versehen.

49 Dazu sei neben dem Überblick in Polonio 2003 bes. auf Bertini Guidetti 1998 verwiesen.

50 Aus der umfangreichen kunsthistorischen Literatur seien hier nur Di Fabio 1998a und Novello 2012 zitiert. 
Diese verlaufen im Hauptschiff oberhalb der Säulenstellung, wobei die eine auf der linken, nördlichen Seite über der vierten Arkade einsetzt und von West nach Ost führt, während die andere, längere, im Süden an der Vierung beginnt und nach Westen verläuft (Abb. 14). Die an der Nordarkade angebrachte, 1307 datierte Inschrift hält die Erneuerung dieses Bauteils durch Vertreter der Kommune aus den Einnahmen des Zehnten fest. ${ }^{51}$ Oberhalb dieser Bauinschrift ist an den Scheinemporen der Nordseite eine Büste angebracht, die wiederum durch eine Inschrift kommentiert ist (Abb. 15, Abb. 16, Abb. 17). Diese, in sechs Zeilen aufgeteilt, identifiziert den in der Büste dargestellten als „Janus, erster König Italiens [...] der Genua gründete zur Zeit Abrahams“. 52 Die zweite Bauinschrift, die das Jahr 1312 trägt, nennt erneut zwei städtische Vertreter, die hier mit ihrer Amtsbezeichnung als „reparatores“ erscheinen, und berichtet wiederum von der Baufinanzierung durch den Zehnten. Sie endet damit jedoch nicht, sondern es schließt sich direkt ein inhaltlich ganz anders ausgerichteter Text an. In ihm werden zwei Fürsten mit Namen Janus genannt: Janus sei, aus Troja flüchtend, auf der Suche nach einem sicheren Ort nach Genua gekommen, welches Janus, erster König Italiens, zur Zeit Abrahams gegründet hatte, und er (der Trojaner) ließ es groß und mächtig werden..$^{33}$

Bei den Inschriften handelt es sich um die früheste monumentale Festschreibung des dann breit tradierten Gründungsmythos' Genuas. ${ }^{54}$ Ein solcher wurde hier erst spät, im letzten Jahrzehnt des 13. Jahrhunderts, in vielgelesenen Werken ausgearbeitet, zunächst durch Jacopo Doria, der den Trojaner Janus als Gründer kennt, dann durch Erzbischof Jacopo da Voragine, der in seiner Chronik gleich drei eponyme Heroen aufführt, wobei der dritte der von den Römern als Gott verehrte Janus war, dessen Kult auch nach Genua gelangte. Als Beleg für den gemeinsamen Ursprung einer in innenpolitischen Auseinandersetzungen zerrütteten Bürgerschaft wurden

51 MCCCVII PASTONUS DE NIGRO ET NICOLAUS DE GOANO FECERUNT RENOVARI HOC OPUS DE DECENO LEGATORUM; Silva 1987, 16 mit Korrekturen wie Gerevini 2015, 206.

52 JAN(US) P(RI)M(US) REX / ITALIE DE P(RO) / GENIE GIGAN / TIU(M) Q(UI) FU(N)DA / VIT JAN(UAM) T(EM)P(O)R(E) / ABRAHE [sic]; Silva 1987, 32.

53 MCCCXII FILIPP(US) D(E) NIGRO (ET) NICOLAU(S) D(E) GOANO REPARATORES HUI(US) ECCL(ES) IE FECER(UN)T RENOVA(R)I HOC OP(US) D(E) D(E)CENO LEGATO(RUM). IAN(US) PRI(N)CEPS TROIAN(US) ASTROLOGIA PERIT(US) NAVIGA(N)DO AD HABITA(N)DU(M) LOCU(M) QUERE(N)S SANU(M) D(OMI)NABILE(M) (ET) SECURU(M), IANUA(M), IA(M) FU(N)DATA(M) A IANO REGE YTALIE P(RO)NEPOTE NOE VENIT ET EA(M) CERNE(N)S MARE (ET) MO(N)TIB(US) TUTISSIMA(M) A(M) PLIAVIT NO(M)I(N)E (ET) POSSE; Silva 1987, 17 mit Korrekturen wie Gerevini 2015, 212. Vgl. zu den Inschriften auch Calderoni Masetti/Wolf 2012, Bd. 1, 365-367 Schede 559, 560, 561 (Stefano Riccioni) und gute Abbildungen der Inschriften ebd., Bd. 2, $536 \mathrm{f}$.

54 Für die Inschriften im Kontext des Genueser Janus-Mythos Di Fabio 1979; Di Fabio 1998b; Beneš 2011 (s. hier für den Vergleich mit den Gründungsmythen anderer Städte); Gerevini 2015 (bes. aus der Perspektive epigraphischer Fragestellungen); für den politischen Hintergrund auch Bertini Guidetti 1998. 


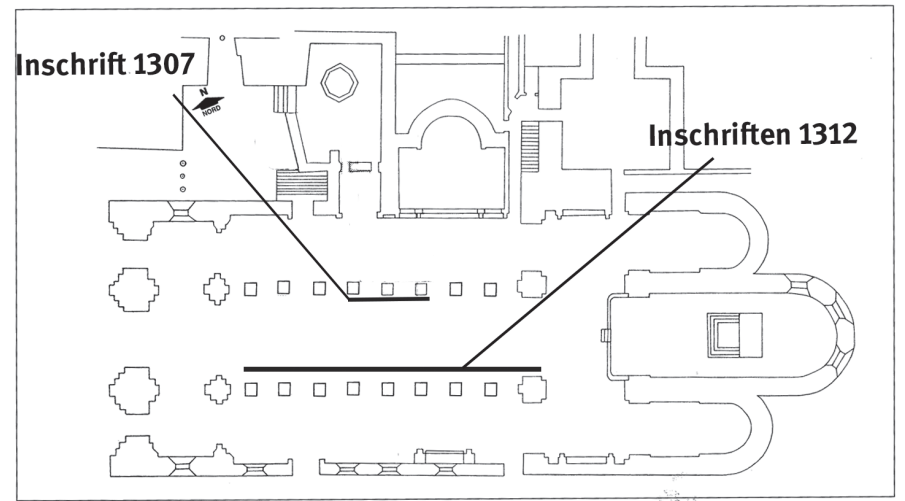

Abb. 14: Grundriss der Kathedrale San Lorenzo mit Lokalisierung der Bauinschriften (aus: Anna Rosa Calderoni Masetti u. Gerhard Wolf (Hgg.) (2012), La cattedrale di San Lorenzo a Genova, Bd. 2 (Mirabilia Italiae 18), Modena, 537 (überarbeitet)).
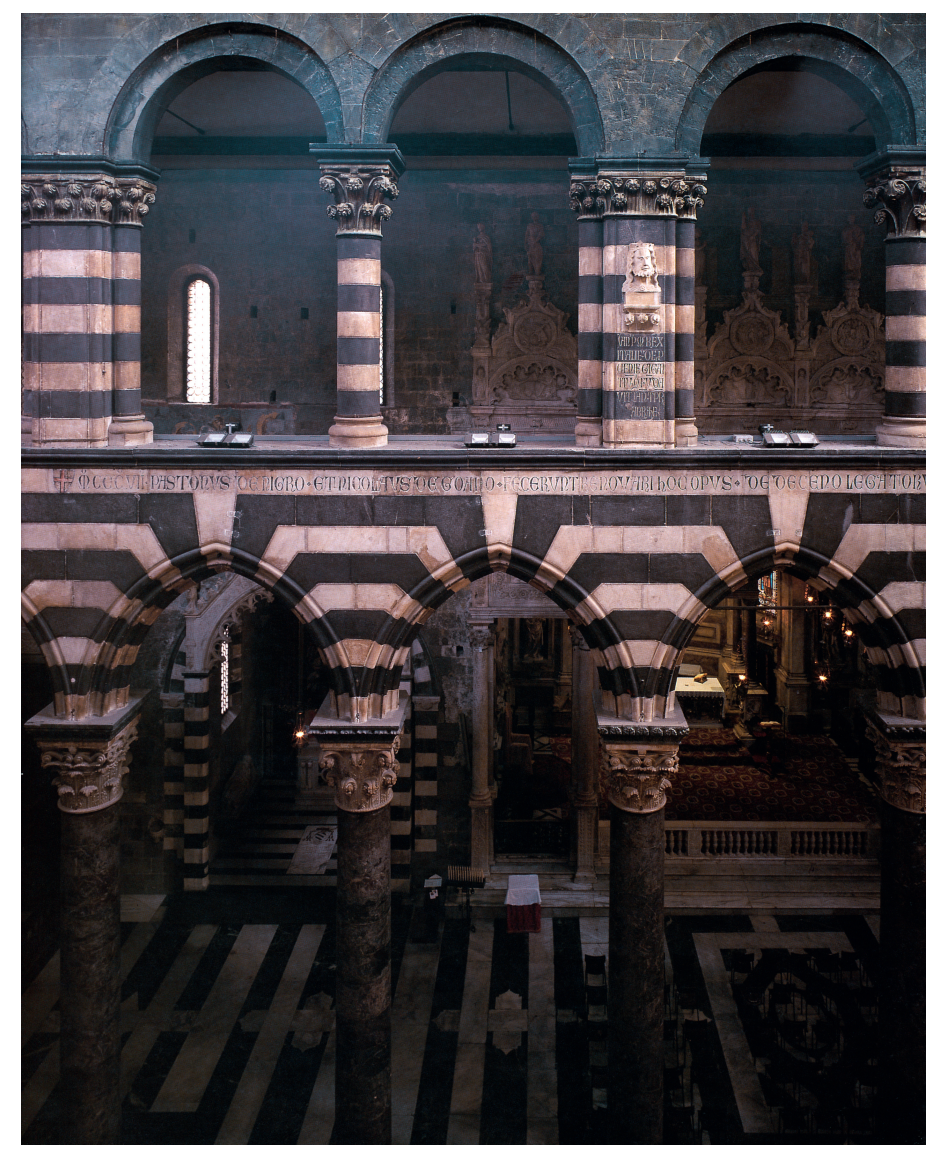

Abb. 15: Genua, San Lorenzo, Blick vom Hauptschiff auf die Nordarkaden (aus: Di Fabio 1998b, 259). 


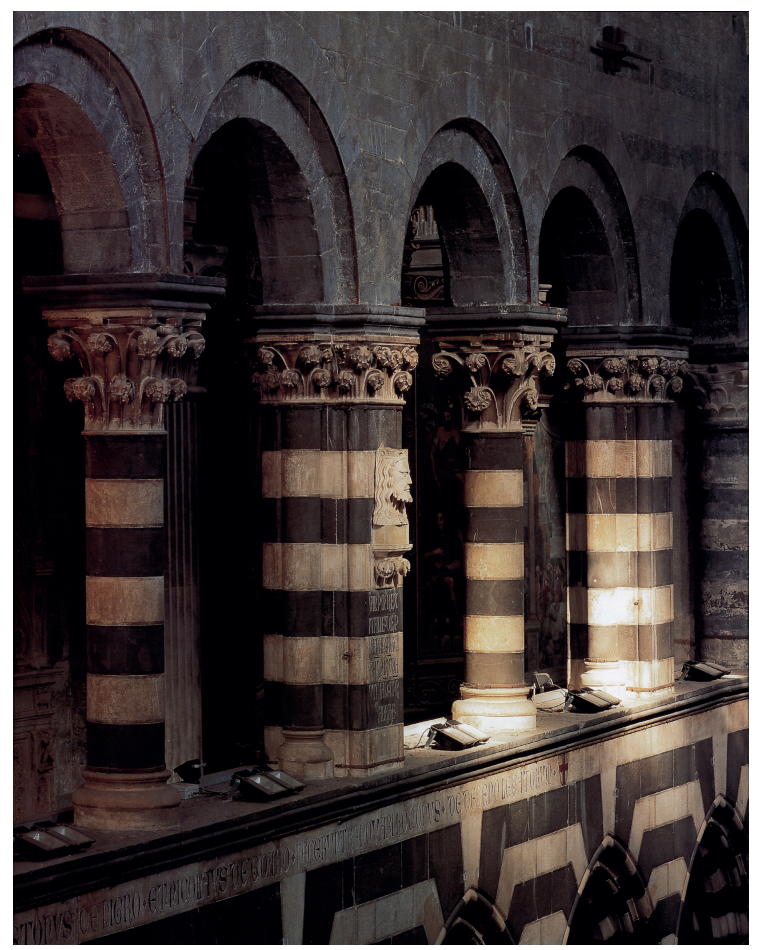

Abb. 16: Genua, San Lorenzo, nördliche Scheinempore mit Inschriften und Büste (aus: Di Fabio 1998a, 225).

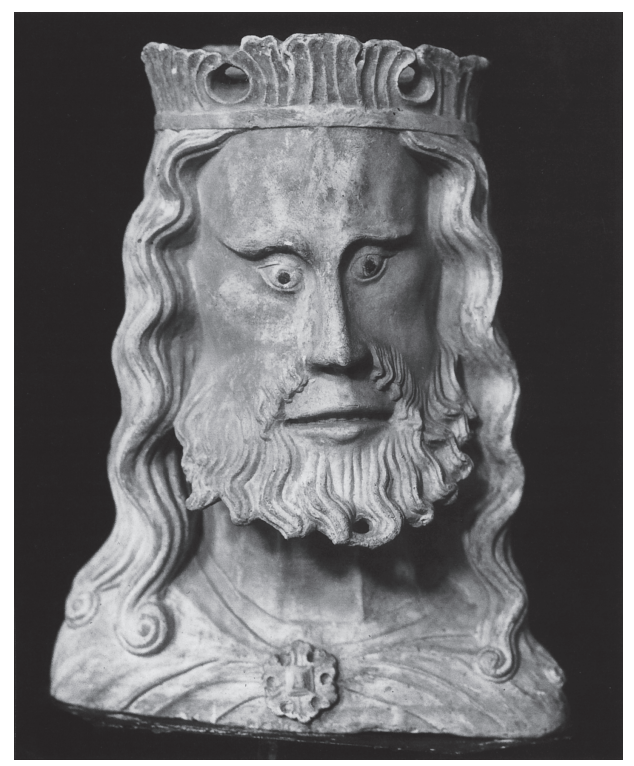

Abb. 17: Genua, San Lorenzo, Büste des „König Janus“, um 1307 (aus: Di Fabio 1998c, 280). 
wichtig: Janus, König Italiens und Gründer Genuas, den die Büste zeigt, und jener zweite, der aus Troja kommend die Stadt zu ihrer Blüte führte.

Die Inschriften in San Lorenzo fallen dadurch auf, dass sie das Innere eines Baues auszeichnen und ihre Wirkung nicht, wie wohl ein Großteil dessen, was als „kommunale Inschrift“ bezeichnet werden kann, am Außenbau entfalten. Stefania Gerevini hat jüngst herausgearbeitet, dass die Inschriften durch ihre Monumentalität und schiere Länge den wichtigsten religiösen wie städtischen Raum, die Kathedrale, an dieser Stelle als einziges Ornament schmücken und als kommunalen Raum markieren, wie dies der Nutzung von San Lorenzo entspricht. Mit ihrem regelmäßigen Layout, wenigen Abkürzungen und großen, eingefärbten Buchstaben waren sie (auch) auf Lesbarkeit hin konzipiert. Die als Faktum formulierten Aussagen zur Gründung der Stadt werden mit den nüchternen Renovierungsinschriften kommunaler Amtsträger verbunden und sind durch das Genueser Wappen eingerahmt. Das Medium der Inschrift vermittelt hier mit dem ihm spezifisch eigenen Potential einmal mehr Autorität und Glaubwürdigkeit.

Welche Funktion und Wirksamkeit kommt in diesem Kontext dem ungewöhnlichen profanen Königsbild zu? Was ist sein „Mehrwert“ gegenüber den Inschriften? Gerevini hat zu Recht betont, wie sehr Bild und Text in ihrer Verortung aufeinander abgestimmt sind, denn Büste und Inschrift sind an der nördlichen Scheinempore genau dort angebracht, wo gegenüber an der südlichen Empore die Bauinschrift endet und nach einem lediglich aus drei Punkten bestehenden Worttrenner der Gründungsmythos beschworen wird. Die Buchstaben werden hier noch größer und lesbarer. Über die Funktion der gewählten Bildform ist damit jedoch noch nichts gesagt.

Die Büste wird einem lombardischen Meister zugeschrieben, der einen Teil des Bauschmucks der Kathedrale schuf, und dem man auch Werke in anderen Kirchen Genuas zugeordnet hat. Der eckige Gesichtsschnitt und die engstehenden mandelförmigen Augen unter breit geführten Brauen finden etwa in den Kapitellen in San Lorenzo selbst Vergleiche, ebenso an zeitgleichen Genueser Bauten wie dem Kreuzgang von San Francesco di Castelletto. ${ }^{55}$ Der schmale Schulterausschnitt und die fehlende Rundung des Hinterkopfes legen nahe, dass die Skulptur nicht aus dem Vollrund reduziert wurde oder ursprünglich einen größeren Ausschnitt zeigte. Es handelt sich nicht um ein vorhandenes wiederverwendetes Stück, wie Teile der älteren Forschung ohne weitere Begründung annahmen, sondern alles spricht dafür, dass die Büste im Kontext der Bauerneuerung für diesen Ort entstand.

55 Grundlegend für eine stilkritische Einordnung Di Fabio 1998c; siehe auch Calderoni Masetti/Wolf 2012, Bd. 1, 367 Scheda 561 (Roberto Paolo Novello) hier zu der Vermutung, dass der Marmorblock, auf dem die Büste aufsitzt, „forse non originale“ sei. Die Datierung der Konsole wird nicht angezweifelt und es gibt keinen Grund für die Annahme, diese habe nicht seit der Erneuerungskampagne die Büste getragen. 
Warum wählte man das Medium der Skulptur, und warum das Format der Büste? Büsten konnten als die antike Darstellungsform par excellence gelten, sowohl für hochgeborene Sterbliche als auch für Götter. Sie blieben an antiken Monumenten sichtbar, etwa am Augustusbogen in Rimini, an Sarkophagen und an Kapitellen. Im 13. und 14. Jahrhundert wurde die Büstenform in der Skulptur für zeitgenössische Bildnisse aktualisiert, etwa durch einzelne Päpste, am Capuaner Brückentor Friedrich II. und im Prager Dom. ${ }^{56}$

In Genua selbst konnte man auf ein Vorbild zurückgreifen. Am Fuß des Kampanile der 1180 durch den Orden vom Hospital des hl. Johannes errichteten Kirche San Giovanni di Prè ist leicht über Kopfhöhe ein einen halben Meter breites, spitzbogiges Marmorrelief mit einer Inschrift vermauert (Abb. 18). Unabhängig von ihrer umstrittenen Transkription kann festgehalten werden, dass sie das Datum des Baubeginns nennt und zur Fürbitte für einen „Guglielmo“ auffordert. ${ }^{57}$ Die jüngere Forschung identifiziert diesen mit einem im Zusammenhang des Hospitals als frater hospitalarius Sancti Johannis und comandator ospitalis erwähnten Mann. Ein nur literarisch überliefertes Grabepitaph bezeichnete diesen Guglielmo als templi fundator. ${ }^{58}$ Die heute noch vorhandene Inschrift rahmt als Spitzbogen das Relief einer männlichen Büste, die - im Profil in zeitgenössischer Haartracht und ohne Heiligenattribute gegeben - als Bildnis des Gründers zu verstehen ist. ${ }^{59}$

Es muss offen bleiben, ob und wie dieses Bild-Text-Ensemble im 14. Jahrhundert, als der Bildhauer die Janusbüste schuf, verstanden wurde. Gleichwohl lässt sich festhalten, dass die Büste im Kontext des Memorialporträts einer Gründerpersönlichkeit in Genua selbst präsent war. Das Büstenmotiv und seine skulpturale Ausprägung erscheinen daher als eine bewusste Wahl für den König Janus. Als ein für eine Emporenwand ungewöhnliches Objekt lenkt die Büste die Aufmerksamkeit auf diese Zone.

56 Zur mittelalterlichen Bildnisbüste Müller 2007.

$5754 \times 35 \mathrm{~cm}$. Ausführlich diskutierte Remondini 1874 die Lesung der Inschrift und transkribierte (ohne Kennzeichnung der Ergänzungen): ACTONIS WILIELMI DOMINI DOMUS EXII HIC. PRO QUO QUESO PATER QUI TRANSIS DIC. MCLXXX TENPORE UILELMI INCOATUM EST; er übersetzte: „Io riuscii qui sepolcro del signor Guglielmo Acton. Per il quale di grazia o tu che passi recita un Pater. Fu principiato nel 1180 al tempo di Guglielmo“ (ebd., 457); Silva 1987, 7 transkribierte ohne Diskussion: ACTOR(E) W(ILIELMO) D(OMI)NI DOM(US) EXII H(IC) / P(RO) Q(U)O QUESO PATER Q(UI) TR(A)NSI(S) D(I)C(I) / MCLXXX TEN(PO)R(E) V(ILIELM)I INCOATU(M) E(S)T. Akzeptiert man diese Lesung, ergibt sich als mögliche Übersetzung: „Ich bin an dieser Stelle hervorgegangen als Haus des Herrn unter der Verwaltung des Guglielmo. Ich bitte Dich, der Du vorbeigehst, sprich ein Vaterunser. 1180, zur Zeit des Guglielmo, wurde (der Bau) begonnen“.

58 Remondini 1878, 27 zitiert nach einem älteren MS des Francesco Maria Accinelli: [...] praeceptor dignus cleri pater ore benignus / templi fundator huiusque domus reparator / hoc iacet in busto Wilielmus more vetusto.

59 Zu dem Relief, das - m. E. auf der Grundlage nur weniger Anhaltspunkte - einem französischen Meister zugeschrieben wird, Di Fabio 1984, 89f.; Dagnino 1992b; Dagnino 2001 (hier auch ausführlicher zur Person des Gründers mit den obigen Quellenzitaten). 


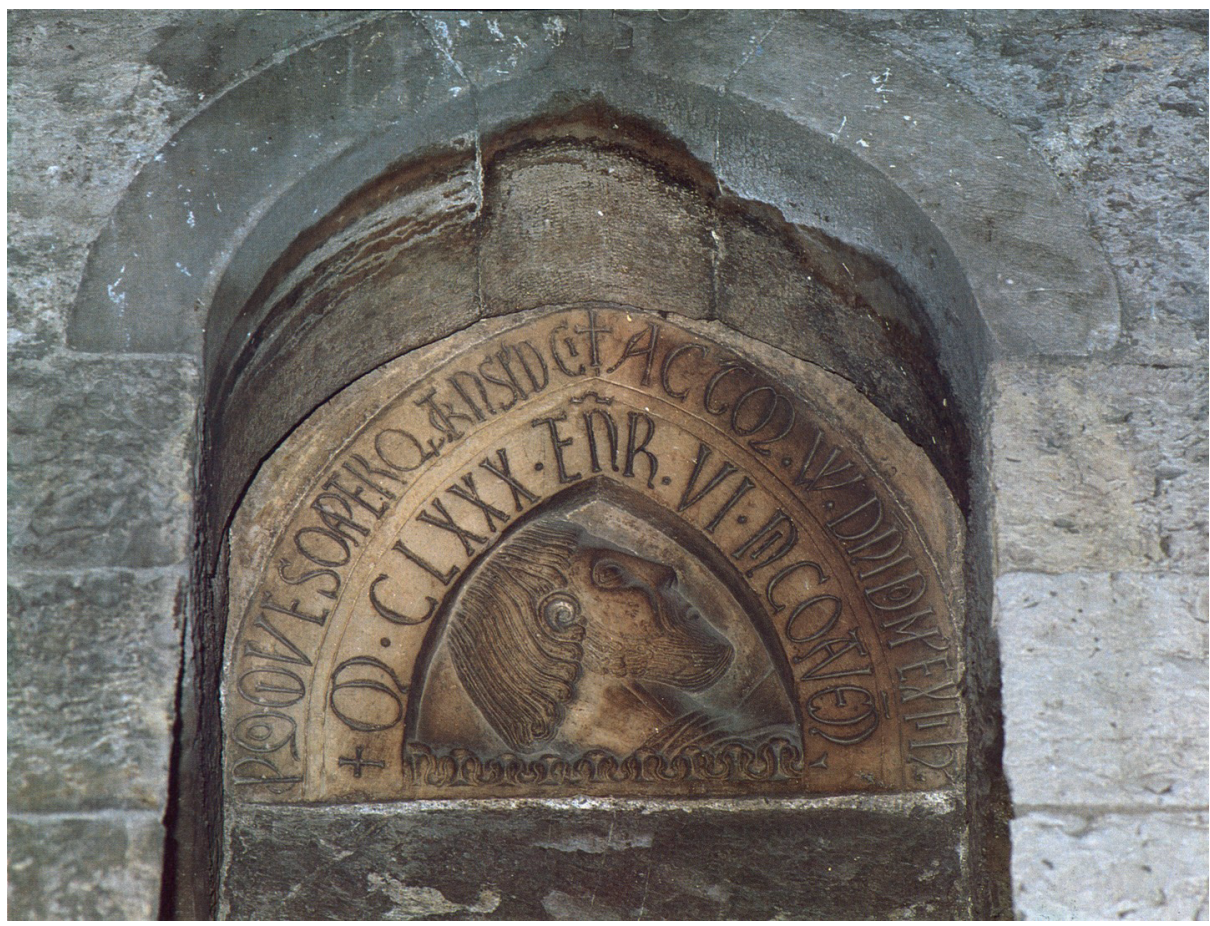

Abb. 18: Genua, San Giovanni di Prè, Relief mit Inschrift und Büstenbildnis zur Erinnerung an den Baubeginn unter frater Guglielmo, 1180 (aus: Clario Di Fabio (Hg.) (1987), La Scultura a Genova e in Liguria, Bd. 1: Dalle Origini al Cinquecento, Genua, 117).

Während sich der Bildhauer in stilistischer Hinsicht in keiner Weise an antiken Vorbildern orientiert, rekurriert er im Bildnisformat auf antike Büsten und ihre Konnotation als einer Präsentationform hervorgehobener Persönlichkeiten oder Götter, wie sie für einen altehrwürdigen Stadtgründer angemessen erscheinen musste. Das Bild war damit geeignet, der in den Inschriften von San Lorenzo vollzogenen Geschichtskonstruktion Anschaulichkeit und Autorität zu verleihen.

Die Diskussion einiger Genueser Erinnerungsmale, die die Bild- und Textmedien verbinden, sollte die unterschiedlichen Konstellationen und Strategien aufzeigen, mit denen das Potential nicht nur der jeweiligen Gattung, sondern ihres dinglichen Zusammenwirkens ausgelotet wird. Dabei wurde deutlich, dass weitere Medien städtischer Autorität und Erinnerung in die Akte des Darstellens und Sprechens eingebunden waren, etwa wenn ein städtisches Siegel zum Relief monumentalisiert und eine erbeutete Inschrift in der Geschichtsschreibung zitiert werden, oder wenn ein Siegel der gegnerischen Kommune, Gegenstand der städtischen Repräsentation par excellence, zur Trophäe wird und dies eine Inschrift proklamiert.

Eine vergleichbare Vielschichtigkeit der Kommunikation hat Marc von der Höh jüngst für Pisa unterstrichen, indem er nicht-schriftliche Medien wie Gefangene und 
die öffentliche Rede einbezog. Es wäre sicher lohnend, auch für Genua der Rolle nachzugehen, welche die Dimension des laut gesprochenen Wortes gerade im Verhältnis zum geschriebenen gespielt haben könnte. ${ }^{60}$ Die Strategien des Zitierens, Verweisens, Wiederaufnehmens lassen nicht nur als Ziel erkennen, Aussagen zu unterstreichen und zu verstärken, sondern diese werden, mit unterschiedlicher Akzentuierung, suggestiv veranschaulicht, differenziert und einem breiteren Kreis über verschiedene Verständnis- und Erlebnisebenen zugänglich gemacht. Das Hafenrelief etwa fordert einen aktiven Betrachter, der auch zu Transferleistungen im Umweg über antike Texte fähig ist.

Möchte man sich den Bedingungen von Wahrnehmung in Genua zumindest annähern, dann muss man sich einerseits vergegenwärtigen, dass die Seh-Erfahrungen des Publikums bemerkenswert breit waren. Für den hier diskutierten Zusammenhang ist vor allem an die Beteiligung eines großen Teils der Genueser Bevölkerung am Fernhandel, an die auswärtigen Kaufleute und an die zeitweise Tausende von Pisanern umfassende „Gemeinde der gefangenen Pisaner“ nach der Meloriaschlacht zu denken. ${ }^{61}$ Wohl nicht zufällig hingen an der Kirche San Torpete, die dieser Gemeinde diente, ebenfalls die Hafenketten, und den Pisanern dürfte die Darstellung ihres stolzen Hafens die Niederlage um so bitterer gemacht haben. Carrie Beneš hat in ihrer Untersuchung von „Urban Legends“ im kommunalen Italien und speziell der Genueser Ianus-Inschriften darauf hingewiesen, dass in Genua Latein besonders lange in Urkunden und Gesetzestexten verwendet und als internationale Handelssprache geläufig war. ${ }^{62}$ Der Anteil der Betrachter, die auch Leser waren oder zumindest eine teilweise Lese- und Verständnisfähigkeit besaßen, wird von ihr daher vergleichsweise hoch eingeschätzt. Zumindest zwei Zeugnisse über das Lesen von Inschriften in Genua sind überliefert, freilich durch Exponenten der intellektuellen Elite: Francesco Petrarca gibt sich als Leser der Inschriften in San Lorenzo zu erkennen, wenn er schreibt, der Janus-Mythos sei nicht nur in den städtischen Chroniken festgehalten, sondern ebenso publicis insculpta monumentis ${ }^{63}$. Der Geschichtsschreiber Giorgio Stella beruft sich für den Wahrheitsgehalt dessen, was er über den Sieg des Lamba Doria berichten kann, auf Inschriften als Quellen, da zu dieser Zeit (1298) kein Annalist tätig und Jacopo Doria bereits verstorben gewesen sei: verum quibusdam notis sic scriptum,

60 von der Höh 2015.

61 Zur Anzahl der am Fernhandel Beteiligten zuletzt van Doosselaere 2009, 79 und Anm. 36; zu den gefangenen Pisanern in Genua Ceccarelli Lemut 1984; für den sozialgeschichtlichen Kontext Jehel 1993, bes. 157-242, 368-432.

62 Beneš 2011, 68.

63 Autorem urbis et nominis Ianum ferunt, primum ut quibusdam placet Italie regem. Quod an ita sit, an ipse situs urbi nomen dederit, quod nostri orbis quasi, ianua' quedam esse videatur, incertum habeo. Prima ibi celebrior opinio est et in chronicis eorum scripta et publicis insculpta monumentis, Petrarca 1990, 42. 
etiam quibusdam locis Janue sculptum inveni. ${ }^{64}$ Bemerkenswert ist bei beiden Quellen, dass der Kontext unmissverständlich deutlich macht, dass es um Texte geht, die zu einer Deutung historischer Ereignisse herangezogen werden. Der Begriff des sculpare deckt jedoch auch ein bildnerisches Arbeiten in Stein mit ab.

Manetti, Petrarca und Stella bieten freilich nur Splitter von Wahrnehmung, die die Präsenz und die Wirkung der diskutierten Monumente reflektieren. Aus produktionsästhetischer Perspektive ist zu betonen, welch großer finanzieller, zeitlicher und wohl auch konzeptioneller Aufwand von einem Teil der Auftraggeber für angemessen und sinnvoll erachtet wurde - die Doria etwa rechneten offenbar mit einer erheblichen Wirkung ihrer Inschriften.

Eine unmittelbare Verbindung von Objekten und Inschriften überwiegend profanen Inhalts wurde in Genua offenbar häufiger als etwa in Pisa oder Venedig umgesetzt, zumindest stellt sich die Überlieferung so dar. Ob wir hierbei tatsächlich von einem besonderen Charakteristikum des so ausgeprägten Genueser „epigraphic habit“ sprechen können, und, wenn ja, weswegen es gerade in dieser Stadt zum Tragen kam, inwieweit das hier nur umrissene Publikum dabei eine Rolle spielte, kann am Ende dieses Beitrags nur als Frage formuliert werden.

\section{Literaturverzeichnis}

Airaldi, Gabriella (2004), Guerrieri e mercanti. Storie del medioevo genovese, Marene.

Alizeri, Federico (1846-1847/1969), Guida artistica per la città di Genova, 3 Bde., Genua 1846-1847, Nachdruck: Bologna 1969.

Belgrano, Luigi Tommaso/Imperiale di Sant'Angelo, Cesare (Hgg.) (1926), Annales lanuenses, dal MCCLI al MCCLXXIX, Genua.

Beneš, Carrie E. (2011), Urban Legends: Civic Identity and the Classical Past in Northern Italy, 1250-1350, University Park.

Bertini Guidetti, Stefania (1998), Potere e Propaganda a Genova nel Duecento, Genua.

Bierman, Irene A. (1998), Writing Signs. The Fatimid Public Text, Berkeley u. a.

Bleumer, Hartmut/Patzold, Steffen (2003), „Wahrnehmungs- und Deutungsmuster in der Kultur des europäischen Mittelalters“, in: Das Mittelalter 8 (2), 4-22.

Bottazzi, Marialuisa (2012), „Città e scrittura epigrafica“, in: Miriam Davide (Hg.), Identità cittadine e aggregazioni sociali in Italia, secoli XI-XV, Triest, 275-302.

Breveglieri, Bruno (1997), „Il volgare nelle scritture esposte bolognesi. Memorie di costruzioni e opere d'arte“, in: Claudio Ciociola (Hg.), „Visibile parlare“. Le scritture esposte nei volgari italiani dal medioevo al rinascimento, Neapel, 73-99.

Calderoni Masetti, Anna Rosa/Wolf, Gerhard (Hgg.) (2012), La Cattedrale di San Lorenzo a Genova / The Cathedral of St Lawrence in Genoa (Mirabilia Italiae 18), 2 Bde., Modena.

Cavallaro, Luisa (1992), Il palazzo del Mare. Il nucleo medioevale di Palazzo San Giorgio, Analisi scientifica e rilievi di Patrizia Traverso, prefazione di Colette Dufour Bozzo, Genua.

64 Giorgio und Giovanni Stella 1975, 36. 
Ceccarelli Lemut, Maria Luisa (1984), „I Pisani prigionieri a Genova dopo la battaglia della Meloria: la tradizione cronistica e le fonti documentarie“, in: Renzo Mazzanti (Hg.), 1284. L'anno della Meloria, Pisa, 75-88.

Costa, Lorenzo (1859/1892), „A Jacopo Doria. Contro la proposta fatta da alcuni di restituire a Pisa le catene del suo porto conquistate dai genovesi nel 1290“ (Oktober 1859), in: Lorenzo Costa, Canzoniere, Genua, 130-134.

Croce, Isabella (2008), „L'ultimo ritratto di Vico Dritto di Ponticello“, in: Ennio Poleggi u. Isabella Croce (Hgg.), Ritratto di Genova nel'400 veduta d'invenzione, Genua, 42-49.

Dagnino, Anna (1987), „Scultura e Architettura“, in: La Scultura a Genova e in Liguria, Bd. 1: Dalle Origini al Cinquecento, Genua, 131-151, (164-169, 173-174).

Dagnino, Anna (1992a), „Rilievo di Porto Pisano“, in: Enrico Castelnuovo (Hg.), Niveo de Marmore. L'uso artistico del marmo di Carrara dall'XI al XV secolo, Genua, 199.

Dagnino, Anna (1992b), „Lapide-ritratto di Guglielmo“, in: Enrico Castelnuovo (Hg.), Niveo de Marmore. L'uso artistico del marmo di Carrara dall'XI al XV secolo, Genua, 155-156.

Dagnino, Anna (2001), „Lapide-ritratto di frate Guglielmo“, in: Giorgio Rossini (Hg.), La Commenda dell'Ordine di Malta arte e restauri di un ospitale genovese del Medioevo, Genua, 198-199.

Debiais, Vincent (2009), Messages de pierre. La lecture des inscriptions dans la commmunication médiévale (XIIIe-XIVe siècle), Turnhout.

Del Punta, Ignazio (2015), La battaglia della Meloria. Il più grande scontro navale del Medioevo, Cagliari.

Depkat, Volker (2003), „Kommunikationsgeschichte zwischen Mediengeschichte und der Geschichte sozialer Kommunikation. Versuch einer konzeptionellen Klärung“, in: Karl-Heinz Spieß (Hg.), Medien der Kommunikation im Mittelalter, Wiesbaden, 9-48.

Di Fabio, Clario (1979), „Il ,mito delle origini“ e il nome di Genova nel medioevo“, in: Bollettino ligustico 31, 37-44.

Di Fabio, Clario (1984), Scultura romanica a Genova (Genova, Museo di Scultura e Architettura ligure di S. Agostino. Studi e ricerche 2), Florenz.

Di Fabio, Clario (1989), „La scultura bronzea a Genova nel Medioevo e il programma decorativo della cattedrale nel primo Trecento“, in: Bollettino dell'Arte 55, 1-43.

Di Fabio, Clario (1998a), „'’'incendio del 1296 e la ,reparatio ecclesie“ fra 1297 e 1317“, in: Clario Di Fabio (Hg.), La cattedrale di Genova nel medioevo secoli VI-XIV, Genua, 223-253.

Di Fabio, Clario (1998b), „Sculture, affreschi ed epigrafi: la città e i suoi ,miti delle origini‘. Fonti, committenti, esecutori“, in: Clario Di Fabio (Hg.), La cattedrale di Genova nel medioevo secoli VI-XIV, Genua, 258-279.

Di Fabio, Clario (1998c), „L'officina della Cattedrale e la scultura a Genova prima di Giovanni Pisano. Un caso di monopolio“, in: Clario Di Fabio (Hg.), La cattedrale di Genova nel medioevo secoli VI-XIV, Genua, 280-299.

Di Fabio, Clario (2007), „Scultura del Duecento in Liguria: materiali e ragionamenti fra ,centro“ e ,periferie““, in: Arturo Calzona et al. (Hgg.), Immagine e Ideologia. Studi in onore di Arturo Carlo Quintavalle, Mailand, 291-301.

Donato, Maria Monica (1997), „Immagini e iscrizioni nell’arte ,politica“ fra Tre e Quattrocento“, in: Claudio Ciociola (Hg.), „Visibile parlare“. Le scritture esposte nei volgari italiani dal medioevo al rinascimento, Neapel, 341-396.

van Doosselaere, Quentin (2009), Commercial Agreements and Social Dynamics in medieval Genoa, Cambridge.

Douët d'Arcq, Louis (1868), Collection de sceaux, Bd. 3, Paris

Ferrando Cabona, Isabella (1998), Palazzo San Giorgio. Pietre, Uomini, Potere, Mailand.

Ferreto de'Ferreti Vicentino (1908), „Historia rerum in Italia gestarum ab anno MCCL ad annum usque MCCCXVIII“, in: Carlo Cipolla (Hg.), Le Opere di Ferreto de'Ferreti Vicentino, Rom. 
Ferretto, Arturo (1904), „Una lapide pisana nel Palazzo di S. Giorgio“, in: Giornale storico e letterario della Liguria 5, 134-142.

Firpo, Marina (2015), I Fieschi. Feudalità e istituzioni. Il Liber privilegiorum (1227-1465), Bd. 1, Genua.

Gamberoni, Francesco (2004), „Uso e iconografia del sigillo comunale a Genova nel medioevo: nuove acquisizioni e alcune precisazioni“, in: Ligures 2, 129-136.

Genova, Pisa e il mediterraneo (1984), Genova, Pisa e il mediterraneo tra due e trecento. Per il VII centenario della battaglia della Meloria (Tagung Genua 1984), Genua.

Gerevini, Stefania (2015), „Written in Stone: Civic memory and Monumental Writing in the Cathedral of San Lorenzo in Genoa“, in: Antony Eastmond (Hg.), Viewing Inscriptions in the Late Antique and Medieval World, New York, 205-229.

Giannozzo Manetti (1974), Elogi dei Genovesi, hg. von Giovanna Petti Balbi, Genua.

Giorgio und Giovanni Stella (1975), Georgii et loannis Stellae Annales Genuenses, hg. von Giovanna Petti Balbi (Rerum Italicarum Scriptores n. s. 17,2), Bologna.

Giovè Marchioli, Nicoletta (1994), „L'epigrafia comunale cittadina“, in: Paolo Cammarosano (Hg.), Le forme della propaganda politica nel Due e nel Trecento (Tagung Triest 1993; Collection de l'École Française de Rome 201), Rom, 263-286.

Hamburger, Jeffrey F. (2014), Script as Image, Paris u. a.

Haug, Henrike (2016), Annales Ianuenses. Orte und Medien des historischen Gedächtnisses im mittelalterlichen Genua, Göttingen.

Herklotz, Ingo (1999), „Antike Denkmäler in den Proömien mittelalterlicher Geschichtsschreiber“, in: Arte d'Occidente: temi e metodi. Studi in onore di Angiola Maria Romanini, 3 Bde., Rom, 971-986.

von der Höh, Marc (2006), Erinnerungskultur und frühe Kommune. Formen und Funktionen des Umgangs mit der Vergangenheit im hochmittelalterlichen Pisa (1050-1150), Berlin 2006.

von der Höh, Marc (2015), „Trophäen und Gefangene. Nicht-schriftliche Erinnerungsmedien im hochmittelalterlichen Pisa“, in: Joachim J. Halbekann, Ellen Widder u. Sabine von Heusinger (Hgg.), Stadt zwischen Erinnerungsbewahrung und Gedächtnisverlust (Tagung Esslingen am Neckar 2010; Stadt in der Geschichte 39), Ostfildern, 147-174.

Jehel, Georges (2003), Les Génois en Méditerranée Occidentale (fin XIème - début XIVème siècle). Ebaude d'une stratégie pour un empire, Paris.

Kedar, Benjamin (2012), „Prolegomena to a World History of Harbour and River Chains“, in: Ruthi Gertwagen u. Elizabeth M. Jeffreys (Hgg.), Shipping, Trade and Crusade in the Medieval Mediterranean: Studies in Honour of John Pryor, Farnham, 3-38.

Kiening, Christian (2007), „Medialität in mediävistischer Perspektive“, in: Poetica 39, 285-352.

Kroll, Renate (2015), „Text-Bild-Beziehungen: Methodische Vorüberlegungen zu einem historischen Streifzug durch die europäische Literatur und Malerei“, in: Renate Kroll, Susanne Gramatzki u. Sebastian Karnatz (Hgg.), Wie Texte und Bilder zusammenfinden, vom Mittelalter bis zur Gegenwart, Berlin, 11-15.

Mathews, Karen R. (2012), „Plunder of war or objects of trade? The reuse and reception of Andalusi objects in medieval Pisa“, in: Journal of Medieval Iberian Studies 4 (2), 233-258.

Mazzanti, Renzo (Hg.) (1984), Mille duecento ottantaquattro, l'anno della Meloria, Pisa.

Müller, Rebecca (2002), Sic hostes Ianua frangit: Spolien und Trophäen im mittelalterlichen Genua, Weimar.

Müller, Rebecca (2005), „Genova vittoriosa. I trofei bellici“, in: Piero Boccardo u. Clario Di Fabio (Hgg.), Genova e l'Europa mediterranea, Mailand, 89-107.

Müller, Rebecca (2007), „Überlegungen zur mittelalterlichen Bildnisbüste“, in: Jeanette Kohl u. Rebecca Müller (Hgg.), Kopf/Bild: Die Büste in Mittelalter und Früher Neuzeit, München, 33-73. 
Müller, Rebecca (2016), „Riflessioni sulla percezione di artefatti islamici nella Genova medievale“, in: Alireza Naser Eslami (Hg.), Genova, una capitale del mediterraneo tra Bisanzio e il mondo islamico. Storia, arte e architettura, Mailand, 107-123.

Novello, Roberto Paolo (2012), „La ricostruzione dopo l'incendio del 1297“, in: Anna Rosa Calderoni Masetti u. Gerhard Wolf (Hgg.), La Cattedrale di San Lorenzo a Genova / The Cathedral of St. Lawrence in Genoa, Bd. 1: Testi / Text (Mirabilia Italiae 18), Modena, 75-82.

Origone, Sandra/Varaldo, Carlo (Hgg.) (1983), Corpus Inscriptionum Medii Aevi Liguriae, Bd. 2: Genova. Museo di S. Agostino, Genua.

Pessa, Loredana (Hg.) (2016), Genova nel Medioevo. Una capitale del Mediterraneo al tempo degli Embriaci, Genua.

Pessa, Loredana (2016), „Tessuti d'oltremare a Genova“, in: Loredana Pessa (Hg.), Genova nel Medioevo. Una capitale del Mediterraneo al tempo degli Embriaci, Genua, 94-103.

Petrarca, Francesco (1990), Itinerario in Terra Santa: 1358, hg. von Francesco Lo Monaco, Bergamo.

Petrucci, Armando (1985), „Potere, spazi urbani, scritture esposte: proposte ed esempi“, in: Culture et idéologie dans la genèse de l'état moderne (Tagung Rom 1984; Collection de l'École Française de Rome 82), Rom, 85-97.

Petrucci, Armando (1986), La scrittura. Ideologia e rappresentazione (Piccola biblioteca Einaudi 473), Turin.

Piaggio, Domenico (MS 1720), Epitaphia, sepulcra et inscriptiones cum stemmatibus, marmorea et lapidea, existentia in ecclesiis genuensibus, Genua, Biblioteca Civica Berio, Bd. 5 (m.r.V.4.5).

Polonio, Valeria (2003), „Da provincia a signora del mare. Secoli VI-XIII“, in: Dino Puncuh (Hg.), Storia di Genova. Mediterraneo, Europa, Atlantico, Genua, 111-232.

Puncuh, Dino (Hg.) (1996), I Libri lurium della Repubblica di Genova, Bd. 1.2, Genua.

Remondini, Marcello (1874), „Di un bassorilievo con iscrizione murato nella torre di San Giovanni di Prè in Genova“, in: Giornale Ligustico 1, 445-460.

Remondini, Marcello (1878), Iscrizioni antichi liguri copiate dal vero ed esposte, Genua.

Rossetti, Gabriella (1989), „Pisa: assetto urbano e infrastruttura portuale“, in: Città portuali del Mediterraneo (Tagung Genua 1985), Genua, 263-286.

Saurma-Jeltsch, Lieselotte E. (2010), „About the Agency of Things, of Objects and Artefacts“, in: Lieselotte E. Saurma-Jeltsch u. Anja Eisenbeiß (Hgg.), The Power of Things and the Flow of Cultural Transformations: Art and Culture between Europe and Asia, Berlin, 10-22.

Schellewald, Barbara (2011), „Einführung. Bild und Text im Mittelalter“, in: Karin Krause u. Barbara Schellewald (Hgg.), Bild und Text im Mittelalter, Köln u. a., 11-21.

Shalem, Avinoam (2015), „What Do Inscriptions on Objects Do? Beyond Calligraphy and Textual History“, in: Amy S. Landau (Hg.), Pearls on a String: Artists, Patrons, and Poets at the Great Islamic Courts, Baltimore, 15-21.

Silva, Augusta (1987), Corpus Inscriptionum Medii Aevi Liguriae, Bd. 3: Genova. Centro Storico, Genua.

Strätling, Susanne/Witte, Georg (2006), „Die Sichtbarkeit der Schrift zwischen Evidenz, Phänomenalität und Ikonizität. Zur Einführung in diesen Band“, in: Susanne Strätling u. Georg Witte (Hgg.), Die Sichtbarkeit der Schrift, München, 7-18.

Ursone da Sestri (1853), „Historia de victoria quam Ianuenses habuerunt contra gentes ab imperatore missas ut subderent sibi lanuam urbem et loca ipsius“, in: Historiae Patriae Monumenta. Chartarum. Bd. 2, Turin, Sp. 1742-1764.

Weber, Christoph Friedrich (2011), Zeichen der Ordnung und des Aufruhrs. Heraldische Symbolik in italienischen Stadtkommunen des Mittelalters, Köln/Weimar/Wien.

Vecchi, Eliana M. (1992), „,Literae marmoreae insculptae‘. Problematiche dell'epigrafia medievale in Lunigiana dall'XI al XIV secolo“, in: Enrico Castelnuovo (Hg.), Niveo de Marmore. L'uso artistico del marmo di Carrara dall'XI al XV secolo, Genua, 294-299. 
Wolf, Gerhard (2015), „Bild, Ding, Kunst. Vierzehn Fallstudien aus Kunstgeschichte und Literaturwissenschaft zu einer begrifflichen Dreiecksbeziehung“, in: Gerhard Wolf (Hg.) unter der Mitarbeit von Kathrin Müller, Bild - Ding - Kunst, Berlin/München, 7-14. 